\title{
III. Weltliterarische Konzepte in der Verlagspraxis
}

\section{III.1. Von ,Weltliteratur' zu ,Literaturen der Welt'}

Nachdem die Problematik weltliterarischer Forschungsperspektiven bis hin zu weltliterarischen Konzepten des Schreibens selbst im Kontext der Besonderheiten einer lateinamerikanischen Literaturgeschichte ausführlich dargestellt worden sind, soll im Folgenden nun die Arbeit an konkreten Beispielen aus der Verlagspraxis im Vordergrund stehen. ${ }^{1}$ Als Ausgangspunkt hilft ein Blick auf die sich derzeit verändernden Kanonisierungsprozesse karibischer Literaturen durch weltweit agierende Verlage ein konzeptionelles Spannungsfeld aufzuzeigen, das sich als grundlegend für die Arbeit am Material darstellt. Im Weiteren dient dann - aufgrund der besonderen Materiallage - das Lateinamerika-Programm des deutschen Suhrkamp Verlags als Rahmen für konkrete Analysen.

Für diesen praxisorientierten Zugang soll nun an eine Begriffsdimension angeknüpft werden, die unter den Terminus ,Literaturen der Welt' gefasst werden kann (vgl. Ette 2004). ${ }^{2}$ Wenngleich ähnliche Bestrebungen auch unter Beibehaltung des Weltliteraturbegriffs unternommen wurden (vgl. etwa die Arbeiten in Küpper 2013), ist das Konzept der ,Literaturen der Welt‘ vor allem als programmatische Modifizierung zu verstehen: Der Begriff knüpft an ,klassische‘ Vorstellungen von Weltliteratur zwar an, doch wird dem alten Begriff eine völlig neue Bedeutung abgewonnen, eine Bedeutung, die unverkennbar jenseits nicht nur des Nationalstaats, sondern jenseits der Nationalliteratur liegt, auch wenn diese weiterhin über äußerst wichtige Instanzen der Produktion, Reproduktion, Distribution und Rezeption verfügt. Die ,Literaturen der Welt‘ haben an Sesshaftigkeit verloren und in zunehmendem Maße nomadisierende, in Bewegung befindliche Denk-, Schreib- und Wahrnehmungsmuster in sich aufgenommen (Ette 2004: 179). Folgende Charakteristika gelten vergleichbar verbindlich für

1 Die Bedeutung materieller Produktions- und Distributionsprozesse als sine qua non der Weltliteratur betont auch Jorge J. Locane in seiner Studie zur Weltliteratur im lateinamerikanischen Kontext: „Así, la literatura mundial, donde, por contraste con las literaturas locales, la cadena de mediaciones y agregado de valor se dilata de modo exponencial, sería el resultado de un complejo sistema de articulaciones y sumatorias [...]. Sin las mediaciones necesarias para acondicionar el artefacto literario y ofrecerlo a esa recepción distante no hay literatura mundial“ (Locane 2019: 216).

2 Dem Spannungsfeld von ,Weltliteratur' und ,Literaturen der Welt' gehe ich auch mit unterschiedlichen Schwerpunktsetzungen in Müller (2014a, 2015a, 2015b, 2017a, 2017d und 2018c) nach.

๖ Open Access. ( 2020 Gesine Müller, publiziert von De Gruyter. (๕)BY-NC-ND Dieses Werk ist lizenziert unter der Creative Commons Attribution-NonCommercial-NoDerivatives 4.0 Lizenz. https://doi.org/10.1515/9783110692174-003 
beide Konzepte, ,Weltliteratur' sowie ,Literaturen der Welt‘, werden aber je nach Perspektivierung oft diametral entgegengesetzt fokussiert:

1) Mehrsprachigkeit: Während für ,Weltliteratur' der Übersetzungsindex ausschlaggebend ist, ist ein häufiges Charakteristikum für ,Literaturen der Welt‘, dass Mehrsprachigkeit in den Romanen inszeniert wird.

2) Bewegung: Die Inszenierung von Bewegung funktioniert beim Konzept ,Weltliteratur‘ immer rückgebunden an eine europäische Perspektive, während ,Literaturen der Welt' für eine Auflösung von Zentrum und Peripherie stehen.

3) Global/Lokal: Die Hinwendung zum Regionalen hat für Befürworter von ,Weltliteratur' meist die Intention, einen Mikrokosmos darzustellen, der stellvertretend für einen Makrokosmos steht, während die konkrete Inszenierung von Regionalität in ,Literaturen der Welt‘ oft das Partikulare betont.

4) Nicht-Sesshaftigkeit: Der Ort des Schreibens ist indirekt, aber nicht zwingend Voraussetzung für die Zuordnung zu einem der beiden Konzepte. Nicht-sesshafte Autor/innen haben privilegierte Voraussetzungen, in den Kanon von ,Literaturen der Welt' aufgenommen zu werden.

5) Deutungshoheit Europa/USA: Beide Konzepte wollen universal verbindliche Literaturen kanonisieren, wobei für ,Weltliteratur ein europäischer Deutungsanspruch behauptet wird. Von Vertretern von ,Literaturen der Welt` wird dieser zwar für obsolet erklärt, aber dennoch - angesichts realer Institutionalisierungsprozesse - in der westlichen beziehungsweise nördlichen Hemisphäre ausgeübt.

Im Umfeld verschiedener Institutionen des Literatur- und Kulturbetriebs, die um eine Perspektiverweiterung bemüht waren, ist der Begriff ,Literaturen der Welt“ etwa seit dem Jahr 2000 aufgekommen: So verfolgt das Haus der Kulturen der Welt in Berlin mit der Pluralbildung einen programmatischen Anspruch von Kultur- und Literaturvermittlung jenseits westlicher Hegemonie, wie auch das Internationale Literaturfestival Berlin, das seit 2001 eine eigene Programmsparte „Literaturen der Welt“ führt. Das Goethe-Institut würdigt mit dem Begriff die besonderen Leistungen der Gesellschaft zur Förderung der Literatur aus Afrika, Asien und Lateinamerika in der Übersetzungsförderung seit den 1970er Jahren und zielt damit auf die Inklusion marginalisierter literarischer Werke und Traditionen in den deutschsprachigen Buchmarkt.

Im universitären Bereich wird der Begriff ab 2002 erstmals diskursiviert, als in Düsseldorf eine Tagung mit dem Titel „Weltpolitik - Weltbewußtsein - Literaturen der Welt" stattfindet, gemeinsam organisiert von Vittoria Borsò und Ottmar Ette. Die Wissenschaften prägen dabei unterschiedliche Begriffe: Während Ottmar Ette (2004, 2013) von ,Literaturen der Welt' spricht und damit eine radikale Abkehr von den in der Weltliteraturdebatte vielfach impliziten Eurozentrismen 
propagiert, verwendet etwa Elke Sturm-Trigonakis (2007) in einem ähnlichen Sinne den Begriff ,Neue Weltliteratur‘. Die Literaturkritikerin Sigrid Löffler, mit klarem Kanonisierungsbestreben auf der Höhe einer globalisierten, von transnationalen Lebensläufen geprägten Zeit, verwendet diese Begriffsbildung für ihre Publikation Die neue Weltliteratur und ihre großen Erzähler (2014) und macht einem größeren, nichtwissenschaftlichen Publikum herausragende Erzähler/innen von Weltrang bekannt: Es sei - so ist auf der Verlagsseite von C.H. Beck zu lesen - eine „völlig neue, nichtwestliche Literatur entstanden, die zumeist von Migranten und Sprachwechslern aus ehemaligen Kolonien und Krisenregionen geschrieben wird. Nomadische Autoren erzählen farbig und prall, reflektiert und in den unterschiedlichsten Tönen Geschichten über gemischte Herkünfte und hybride Identitäten, transnationale Wanderungen und schwierige Integrationen.“3 Mag es sich bei der Zuschreibung „farbig und prall“ um eine in Kontexten wie dem indischen oder dem lateinamerikanischen sehr wohlbekannte Exotisierung von Literaturen handeln, so zeigt sich doch hier: Die Wichtigkeit eines Perspektivwechsels in der Weltliteraturdebatte ist auch im Literaturbetrieb präsent.

Drei Dimensionen dieses Paradigmenwechsels scheinen von besonderer Bedeutung:

a) Die Abkehr von eurozentrischer Begrifflichkeit und eurozentrischem Kanon,

b) der Fokus auf konkrete Entstehungsbedingungen für Kanonisierung in der derzeitigen Globalisierungsphase und unter Berücksichtigung konkreter Übersetzungsprozesse beziehungsweise Verlagspolitiken und

c) eine inhaltlich, das heißt Themen und Autorenbiographien betreffend neu gelagerte Strömung in den Literaturen unserer Zeit auf inter- und transnationaler Ebene.

Der Terminus ,Literaturen der Welt‘ mag diese Dimensionen besonders gut einfangen, da er sowohl eine - wenn auch längst nicht abgeschlossene - wissenschaftliche Begriffsfindung anzeigt als auch eine gängige Praxis im Literaturbetrieb aufgreift. In Literaturkritik und Verlagswesen werden ,Weltliteratur' und ,Literaturen der Welt' schließlich meist in verschiedenen, deutlich voneinander abgrenzbaren Kontexten verwendet: Impliziert ,Weltliteratur' noch immer die unumstößliche Bedeutsamkeit eines Werkes und fördert so den Absatz eines Titels oder unterstreicht die Bedeutung einer Besprechung zu einem Autor/einer Autorin, so meint ,Literaturen der Welt' in der Verlagssprache häufig mehr eine zeitgemäße, neu ausgerichtete Literatur aus weniger bekannten, aber gerade deswegen interessanten Weltgegenden von bedeutendem, aber noch nicht ganz ins

3 https://www.chbeck.de/loeffler-neue-weltliteratur/product/12403092. 
Bewusstsein der Leser gerücktem Rang. Mitgedacht wird dabei eine größere Materialfülle, größere Unübersichtlichkeit, ein Nebeneinander vieler Werke und Traditionen und eine größere Offenheit gegenüber unabgeschlossenen oder kritikwürdigen Selektionsprozessen.

\section{III.1.1. Im Spannungsfeld verlegerischer Konzepte: karibische Literaturen als Beispiel}

Anschließend an erste konzeptionelle Überlegungen zu den Begriffen ,Weltliteratur' und ,Literaturen der Welt‘ lohnt sich ein vertiefender Blick auf die Literaturen der Karibik und hier insbesondere auch auf die Literaturproduktion der französischen Antillen und ihre internationale Rezeption. ${ }^{4}$ Denn karibische Literaturen stehen wie kaum eine andere literarische Tradition für das Zusammenspiel von Deterritorialisierung und topographischer Konkretisierung, für transkulturelle Translations- und Transformationsprozesse von Epistemen und Ästhetiken und für literarische Modellierungen transterritorialer Vernetzungen. ${ }^{5}$ Sie tragen damit den formulierten Kriterien für ,Literaturen der Welt' in überdurchschnittlicher Weise Rechnung. Gleichzeitig sind karibische Literaturen in der Vergangenheit nur sehr unzureichend als ,Weltliteratur' in Betracht gezogen worden. In diesem Spannungsfeld lässt sich auf der praktischen Ebene verlegerischer Selektion eine neu zu konstatierende Wechselwirkung beider Konzepte feststellen: Wenn sie für zwei Möglichkeiten von Paradigmenbildung in Literaturtheorie und Kulturbetrieb stehen und ihre Kriterien oftmals eine große Schnittmenge in der Auswahl literarischer Primärtexte haben, dann zeigt sich eben diese Schnittmenge in besonderer Weise in der internationalen Vermarktung und Rezeption karibischer Literaturen. Genauer gesagt: Die westlich dominierte Verlagsindustrie legt bei der Auswahl internationaler Literaturen aus Regionen, die einst als peripher bezeichnet wurden, in einer ersten Stufe den Selektionsfilter ,Literaturen der Welt' an. Literarische Texte, die dieses Nadelöhr erfolgreich passiert haben, werden in einem zweiten Schritt zu ,Weltliteratur‘.

Beispiele aus der englischsprachigen Karibik illustrieren das Phänomen einer großen Schnittmenge beider Konzepte: Bereits tradierte ,Weltliteratur a as der anglophonen Karibik zeigt die Relevanz der für das Konzept der ,Literaturen der

4 Vgl. dazu auch Müller (2017a, 2017d).

5 Darauf hat u.a. auch Birgit Neumann in unserem gemeinsamen Beitrag zu Vittoria Borsòs Band „Weltliteratur“ hingewiesen, der in der Reihe Grundbegriffe der Literaturwissenschaft im Walter de Gruyter Verlag erscheinen wird. 
Welt' neu formulierten Kriterien der Nicht-Sesshaftigkeit, der Inszenierung von Bewegung in Ablösung von Zentrum-Peripherie-Logiken und einer Betonung des Partikularen, oftmals auch des Archipelischen. Offenbar sind gerade solche Texte aus der englischsprachigen Karibik als ,Weltliteratur' kanonisiert worden, die dem genannten Perspektivwechsel hin zu ,Literaturen der Welt‘ entsprechen können. Man denke nur an Derek Walcotts postkoloniales Epos Omeros aus dem Jahre 1990 (dt. 1995), ein ,in vielerlei Hinsicht [...] paradigmatisches weltliterarisches Werk - und dies weniger wegen seiner Übersetzung in andere Sprachen als vielmehr wegen seiner literarisch vollzogenen Transfer- und Transformationsgeschichten, die es zu einem vielschichtigen Experimentierraum für ein geteiltes, aber lokal situiertes Weltbewusstsein machen“ (Müller/Neumann im Druck).

Im Kontext von Weltliteraturlogiken und Kanonisierungsprozessen spielt es eine entscheidende Rolle, wenn Texte nicht erst in die nach wie vor wichtigste Sprache für globale Zirkulationsprozesse, ins Englische, übersetzt werden müssen, weshalb die englischsprachige Karibik hier eine Sonderstellung einnimmt. Erwähnenswerte Texte, die erfolgreich weltliterarisch kanonisiert wurden, sind neben Walcotts Omeros beispielsweise Edward Kamau Brathwaites The Arrivants (1967-1973), Jean Rhys' Wide Sargasso Sea (1966), V.S. Naipauls The Enigma of Arrival (1987) und Dionne Brands In Another Place, Not Here (1996). Während Literaturen aus der englischsprachigen Karibik also durchaus als ,Weltliteratur“ rezipiert worden sind und gleichzeitig richtungsweisend für Konzepte von ,Literaturen der Welt' sein können, ist eine weltliterarische Kanonisierung im Falle der spanisch- und insbesondere der französischsprachigen Karibik gar nicht oder nur in Ausnahmefällen erfolgt, von denen ich drei schlaglichtartig vorstellen möchte: 1. Unstrittig zum Kanon der Weltliteratur gehört für die erste Hälfte des 20. Jahrhunderts der auf Guadeloupe geborene und aufgewachsene Saint-John Perse mit seinen Dichtungen (Eloges 1911; Anabase 1924; Exil 1942; Vents 1946; Amers 1957), die sich gerade nicht an der karibischen Lebenswirklichkeit orientieren, sondern an der modernen französischen Lyrik in der Tradition Arthur Rimbauds. 1960 erhielt Saint-John Perse den Nobelpreis für Literatur.

2. In die in den 1930er Jahren erstarkende, ursprünglich frankophon geprägte Négritude-Bewegung mit ihrem neuen ,schwarzen' Selbstbewusstsein finden auch Autor/innen der hispanophonen Karibik Eingang. Von besonderer durchaus weltliterarischer - Bedeutung ist hier der kubanische Autor Alejo Carpentier. Seine Werke sind einer eigenen Variante des real maravilloso zuzuordnen. Der Begriff des Magischen Realismus, für den lateinamerikanische Autor/innen später weltberühmt wurden, geht auf eine Formulierung im Vorwort seines Romans El reino de este mundo (1949) zurück. Selektionsfilter für eine besonders günstige Aufnahme der literarischen Qualität von Carpentiers Werk sind seine Anschlussfähigkeit an den französischen Surrealismus und 
eine spezifische Art der Realitätsdarstellung, die mythisch-magische Praktiken als real inszeniert und beim europäischen Publikum als exotistisch anklang.

3. Kubanische Literaturen insgesamt hatten seit der Kubanischen Revolution von 1959 bis in die späten 1970er Jahre hinein eine Modellfunktion, die durchaus auf einer weltliterarischen Ebene rezipiert und in der europäischen und US-amerikanischen Rezeptionslandschaft von führenden Verlagen mit intellektualistischem Anspruch affirmiert wurde. Dies hängt mit dem Boom lateinamerikanischer Literaturen der 1960er Jahre zusammen, wie in Kapitel II.1 ausgeführt. Als knappes Beispiel zu Kuba sei Guillermo Cabrera Infante genannt, der seine literarische Durchsetzungskraft über eine politische (wenn auch anti-kubanische) Dimension sowie über die Anschlussfähigkeit an experimentelle Schreibverfahren entfaltet, die insbesondere im französischen Nouveau Roman vorgeprägt waren.

Während die erwähnten Autoren vor allem im Rahmen einer recht spezialisierten Leserschicht und in ihrer Anschlussfähigkeit an europäische, insbesondere französische Traditionen auf eine gewisse Rezeption verweisen können, hat sich bekanntlich spätestens seit Beginn der 1990er Jahre weltweit eine Gruppe frankophoner Schriftsteller/innen durchgesetzt, die neben der literarischen Produktion auf ein philosophisch motiviertes und aus dem karibischen Erfahrungskontext gespeistes essayistisches Oeuvre verweisen kann. Das Anliegen der Autor/innen um Patrick Chamoiseau und Raphaël Confiant aus Martinique kann als eine Variante der konzeptionellen Implikation von Mehrsprachigkeit in literarischen Texten gelesen werden, wie sie zu Beginn dieses Kapitels als Merkmal von Literaturen der Welt benannt wurde: Mit ihrer Aufwertung oraler kreolischer Traditionen knüpfen sie den Akt der Auflehnung gegen die kulturelle Assimilation, der ein wesentliches Element der literarischen Debatte der Antillen darstellt, nicht an den Inhalt, sondern an die ästhetische Ebene. Der Erfolg dieser literarischen Sprache hat dabei möglicherweise Konsequenzen für das Standardfranzösisch, denn über den Weg der großen Literaturpreise - spätestens seit der Verleihung des Prix Goncourt an Patrick Chamoiseau für seinen Roman Texaco (1992) - gehen Texte in kreolisch-oral durchsetztem Französisch vermehrt in einen sich neu konturierenden literarischen Kanon ein, der in seiner traditionellen Form Grundlage für das Schriftfranzösisch ist. Das Jahr 1992, als der prestigeträchtige Prix Goncourt nach etwas mehr als siebzig Jahren erstmals wieder an einen Autor von den Antillen ging, war sicherlich ein Schlüsseljahr. 1921 hatte zuletzt René Maran mit dem Roman Batouala gewonnen, ein Ereignis, das in die Geschichte einging als das erste Mal, dass ein als ,schwarz' deklarierter Autor einen renommierten französischen Literaturpreis bekam. Nach einer der Literaturproduktion auf den 
Antillen nicht angemessen langen Pause erhielt den Preis 1992 also Patrick Chamoiseau für Texaco, einen Roman, der paradigmatisch für das Konzept von Literaturen der Welt stehen kann: Der Text spielt zunächst am Rand von Martiniques Hauptstadt Fort-de-France, wo auf dem ehemaligen Gelände der Mineralölkompagnie Texaco eine banlieue aus improvisierten Wohnungen gewachsen ist, ein illegales Viertel, das von Bulldozern abgerissen werden soll. Der Roman lebt von den Stimmen einer Vielzahl von Figuren, die die koloniale Geschichte Martiniques lebendig werden lassen und so das Verflochtensein von afrikanischen, europäischen und asiatischen Einflüssen in Martinique abbilden. Ein Prix Goncourt ging danach nicht mehr an eine/n karibische/n Autor/in, wohl aber der Prix Médicis, mit dem 2009 der in Montreal verlegte Dany Laferrière für L'enigme $d u$ retour ausgezeichnet wurde, was für die karibische Literaturproduktion und ihre internationale Wahrnehmung wiederum als Schlüsselmoment gelten kann. Von einem exterioren Blick geprägt, erzählt der Text eine Familiengeschichte zwischen Haiti und Kanada, in welcher der Protagonist, ein haitianischer Schriftsteller im Exil, anlässlich der Beerdigung seines Vaters nach Haiti zurückkehrt.

Solche Auszeichnungen sind noch heute Ausnahmeerscheinungen. Es lässt sich aber beobachten, dass der Verlag Gallimard, der zunächst Édouard Glissant, Raphaël Confiant und Patrick Chamoiseau publiziert hat, zunehmend Autor/innen aus der frankophonen Karibik vertritt, nachdem lange auch dort unter den antillanischen etwa kubanische Autor/innen wie der vorhin als Schlaglicht genannte Alejo Carpentier vorherrschten. Als Initialzündung könnte hier das 2007 bei Gallimard publizierte und vielzitierte Manifest Pour une littérature-monde gelten, an dem zahlreiche namhafte Autor/innen wie Maryse Condé, Édouard Glissant, Fabienne Kanor und Dany Laferrière beteiligt waren und das sich dezidiert gegen die Zentrum-Peripherie-Logik des Frankophonie-Konzeptes wendet. Entscheidend ist außerdem, dass diese Gallimard-Autor/innen der frankophonen Karibik sehr stark die Kriterien von ,Literaturen der Welt‘ vertreten.

Während also karibische Literaturen privilegiert als ,Literaturen der Welt‘ gelten können, zeichnet sich zugleich angesichts dieser kurzen Bestandsaufnahme bei Gallimard, möglicherweise auch weit darüber hinaus, ein sich zunehmend etablierendes Zusammenspiel zwischen dem weltliterarischen Kriterium des Übersetzungsindex und,Literaturen der Welt' auf der Ebene verlegerischer Kanonisierungen ab: Was für Literaturen der anglophonen Karibik schon länger gilt, kann man zur Zeit ${ }^{6}$ auch auf dem französischen Buchmarkt beobachten, nämlich dass das Kriterium ,Literaturen der Welt‘ bei der Auswahl karibischer Autor/innen für die Verlagsindustrie immer wichtiger wird. Literarische Texte, die

6 Zeitpunkt dieser Feststellung März 2017. 
das Nadelöhr dieses ersten Selektionsfilters erfolgreich passiert haben, durchlaufen dann in einem zweiten Schritt die Instanzen, die für eine internationale Kanonisierung nach wie vor insbesondere in der westlichen Welt, in unserem Fall in Frankreich, liegen. ${ }^{7}$

Das Beispiel karibischer Literaturen lässt einen Einblick in Veränderungsprozesse zu, die in der Verlagspraxis im Hinblick auf konzeptionelle Spannungsfelder im Rahmen der Debatte um Weltliteratur zu beobachten sind. Um der Frage, wie Weltliteratur eigentlich gemacht wird, auf der Ebene konkreten Materials weiter nachgehen zu können, soll nun das Fallbeispiel eines Verlages in den Blick genommen werden: Wie kommt es überhaupt zu konzeptionellen Ausrichtungen eines Verlagsprogramms in der Weltliteraturfrage? Inwiefern ist verlegerische Programmatik eruierbar? Inwiefern lassen sich vor allem latente Selektionsmechanismen herausdestillieren?

\section{III.2. Fallbeispiel: Lateinamerikanische Literaturen im Suhrkamp Verlag}

Der Suhrkamp Verlag war in der zweiten Hälfte des 20. Jahrhunderts die entscheidende Instanz für die Verbreitung lateinamerikanischer Literaturen in Deutschland. Als einer der zentralen, global agierenden Verlage in Europa wurde er hier auch wegen der besonderen Materiallage als Fallbeispiel gewählt: In ungewöhnlicher Weise wurden bei Suhrkamp über Jahrzehnte hinweg sehr detailliert Verlagsmaterialien archiviert, aus denen die folgenden Ausführungen schöpfen. Anhand dieses Materials lässt sich zeigen, dass dem Verlagsprogramm des Suhrkamp Verlages ein unausgesprochenes Weltliteraturkonzept zugrunde liegt, das die Kriterien bei der Auswahl fremdsprachiger Literaturen prägt.

Wie also hängt die Geschichte der Rezeption lateinamerikanischer Literaturen in Deutschland, in der der Suhrkamp Verlag bis heute mit seinen knapp vierhundert Titeln die Protagonistenrolle innerhalb der Verlagslandschaft einnimmt, mit einer bestimmten Auffassung von Weltliteratur zusammen? Der 2002 verstorbene Verlagschef Siegfried Unseld, der die Geschichte des Verlags über vierzig Jahre geprägt hatte, verschrieb die Auswahlmechanismen seines internationalen Literaturprogramms der Idee des Gründungsvaters des einstigen Insel Verlags

7 Dieser Befund einer gesteigerten internationalen Zirkulation von Literaturen der Welt aufgrund konkreter Verlagspolitiken ist eine Beobachtung, deren systematische Untersuchung noch aussteht. 
Anton Kippenberg: Weltliteratur als eine Biblioteca Mundi, die auf universalen Qualitätskategorien beruhen sollte, war die Devise. Inwiefern sich zu diesem Auswahlkriterium der gerade rezeptionstheoretisch schwer fassbaren Idee des Universalen auch die Idee des Exotischen gesellte, mögen die folgenden Überlegungen zeigen. Zunächst ist entscheidend, dass die Aushandlung des Konzepts Weltliteratur vor einem theoretischen Hintergrund der Betrachtung von Transferprozessen (siehe Werner/Zimmermann 2002) unter maßgeblicher Berücksichtigung nicht-edierter Verlag-Autoren-Korrespondenzen nachgezeichnet und analysiert wird.

\section{III.2.1. Materialbasis: Das Siegfried Unseld Archiv im Deutschen Literaturarchiv Marbach}

Im Dezember 2009 ging der gesamte Nachlass des Suhrkamp Verlags, der in den Worten George Steiners so etwas wie eine internationale Suhrkamp Culture als weltliterarisches Gütesiegel prägte, an das Deutsche Literaturarchiv in Marbach (DLA) und vergrößerte dabei das Marbacher Gesamtarchiv um ein Viertel (vgl. Bürger 2010: 20). Für 2010 war der Wechsel des Verlagshauses von Frankfurt am Main nach Berlin geplant, und dieser Umzug war der äußere Anlass für den Verkauf der Dokumente aus dem Frankfurter ,Tresor‘ - dem Raum im Keller des Verlagshauses in Frankfurt, in dem Geschäftsunterlagen und Autorenpost lagerten (Cammann 2010). Von mehr als 2100 Umzugskisten aus dem Verlag war die Rede; zusammen mit den Unterlagen aus der Villa des Verlegers Siegfried Unseld umfasst der Nachlass mehr als 20.000 Aktenordner und über 25.000 Bücher der Verlage Suhrkamp und Insel, die nach Marbach transportiert wurden. Dieser Bestand „spiegelt Unselds Ziele, Strategien und seinen enormen Einfluss noch deutlicher wider als seine Aufsätze, Bücher und Reden“ (Bürger 2010: 16).

Der Siegfried Unseld-Nachlass gilt als wertvollster Ankauf in der Geschichte des Deutschen Literaturarchivs in Marbach, dessen Herzstück bislang die Sammlung des Cotta Verlages gewesen war, der publizistischen Heimat von Goethe und Schiller. Seitdem nun wird das Siegfried Unseld Archiv (SUA) in Marbach nach und nach der Forschung zugänglich gemacht. Ulrich Raulff, der damalige Leiter des Deutschen Literaturarchivs, sprach der Presse gegenüber von einem Glücksfall, der einem nur einmal in hundert Jahren widerfahre. Aufgrund einer „hohen Ablagemoral“ (Raulff, zit. nach Cammann 2010) im Verlag findet man neben der Korrespondenz mit Suhrkamp-Autor/innen wie Samuel Beckett, Jürgen Habermas oder Julio Cortázar auch interne Berichte und Lektoratsgutachten, Protokolle der Lektoratsversammlungen, aufschlussreiche Reiseberichte u.a. Unselds selbst, sowie Akten der Werbung und der Lizenzabteilung (vgl. Bürger 2010: 17). 
Gerade auch aus lateinamerikanistischer Sicht handelt es sich um ungewöhnlich wertvolles Material, weil der Suhrkamp Verlag in der internationalen Verlagswelt hinsichtlich lateinamerikanischer Literatur eine so entscheidende Rolle gespielt hat. Erstmals in Deutschland ist damit sehr umfassendes Material zugänglich, anhand dessen ein Zeitpunkt vor der literarischen Übersetzung und der Veröffentlichung lateinamerikanischer Autor/innen in Deutschland in den Blick genommen werden kann. Selektionsprozesse etwa lassen sich auf eine so konkrete Weise untersuchen, wie es im deutschen Verlagswesen bezüglich lateinamerikanischer Literaturen bisher nicht annähernd möglich war. Jan Bürger beschreibt das Archiv und seinen besonderen Wert in seinem Kommentar zur Übernahme des Unseld-Nachlasses durch das Marbacher Literaturarchiv wie folgt:

Die Aufzeichnungen über seine [Unselds] erste Begegnung mit Octavio Paz führen exemplarisch vor, wie sorgfältig er bei seiner täglichen Arbeit nicht nur an die von dem verehrten Dichter favorisierte Gegenwart dachte, sondern auch an die Nachwelt. Alle Briefe der Verlagsleitung wurden aufgehoben und systematisch von Unselds Mitarbeiterinnen (allen voran die legendäre Burgel Zeeh) abgelegt, ebenso wie die Notizen und Protokolle. Jede Reise wurde in Berichten dokumentiert [...]. Dies alles hat dazu geführt, dass das Archiv mit ungewöhnlich wenigen Lücken überliefert ist. (Bürger 2010: 17)

Natürlich ist die Materialdecke zu den einzelnen Autor/innen auch in diesem, im Verlag über Jahrzehnte so sorgfältig vorbereiteten und zusammengestellten Archiv unterschiedlich dick. Für diese Studie wurde die Materiallage im Detail berücksichtigt, das heißt es wurden lateinamerikanische Autor/innen ausgewählt, zu denen für die Weltliteraturfrage aufschlussreiches Material tatsächlich vorliegt beziehungsweise zugänglich ist. Soweit die institutionellen Voraussetzungen der Materialbasis: Doch wie hängen internationale Selektionsmechanismen des Verlags mit einer möglichen Idee von Weltliteratur zusammen?

\section{III.2.2. Auswahl internationaler Literaturen bei Suhrkamp: Vorgeschichte mit Samuel Beckett}

In der ersten Phase einer internationalen Programmbildung, das heißt zwischen Anfang der 1950er und Mitte der 1960er Jahre, fällt der Blick im Rahmen einer weltliterarischen Erfolgsgeschichte von Suhrkamp auf Samuel Beckett, dessen Werk wie kein anderes dem von George Steiner 1973 geprägten Begriff der Exterritorialität als konstitutivem Element einer Suhrkamp Culture entspricht. Dies zeigt sich beispielhaft in einer bekannten Passage aus Becketts Molloy (1951), bei der von der Kritik häufig auf eine „zweifache Stimmführung“ aufmerksam gemacht 
wurde, die nie eindeutig auf einen Sprecher zurückgeführt werden könne und über die sich das schreibende Ich selbst nicht im Klaren ist (Weber 2010: 101, 110): „J'avais oublié qui j'étais (il y avait de quoi) et parlé de moi comme j'aurais parlé d'un autre, s'il m'avait fallu absolument parler d'un autre. Oui, cela m'arrive et cela m'arrivera encore d'oublier qui je suis et d'évoluer devant moi à la manière d'un étranger“ (Beckett 1982: 55).

Im Fall Beckett lässt sich exemplarisch beobachten, wie sehr der Publikationserfolg von bestimmten Konstellationen im verlegerischen Feld abhing. Man denke nur an Becketts Niederlagen vor dem Ersten Weltkrieg bei dem Versuch, sein Werk zu publizieren, und an seinen Erfolg danach, als er endlich auf verlegerisches Interesse für das Experimentelle seiner Literatur stieß, und auf Verleger wie John Calder in London, Barney Rosset (Grove Press) in New York oder eben auf Peter Suhrkamp und Siegfried Unseld bei Suhrkamp in Frankfurt. ${ }^{8}$ Diese Verlagshäuser, allesamt in den 1950er Jahren etabliert, wollten eine neue Ästhetik fördern und waren Becketts Literatur gegenüber aufgeschlossen, während sich die bereits länger etablierten Verlage weiterhin sträubten (vgl. Nixon 2011: 4f.).

1953, während eines Paris-Aufenthalts, besuchte Peter Suhrkamp die erste Aufführung von Warten auf Godot (Hartel 2011: 131). Im selben Jahr vereinbarte er mit Jérôme Lindon von Éditions de Minuit, dem französischen Verleger Becketts, dass Suhrkamp die Rechte an der deutschen Übersetzung des Stückes erhalten sollte. Theodor W. Adorno äußerte sich 1959 positiv über Suhrkamps Einsatz und Weitsicht ob des Erfolgs avantgardistischer Kunst: Suhrkamp gelinge es, das Unverkäufliche zu verkaufen, denjenigen Erfolg zu verschaffen, die nicht danach strebten, und dem Publikum die ,schwer Verwertbaren' nahe zu bringen (Hartel 2011: 132). Tatsächlich stießen Becketts Werke im Nachkriegsdeutschland intellektuelle Debatten in der gesamten Gesellschaft an (Huber 1991: 31).

Eckart Voigts-Virchow beschreibt Beckett als transeuropäischen Autor, weil er die Grenzen nationaler Literaturen transzendiere (2009: 97). Die BeckettForschung hat darauf hingewiesen, dass Unseld diese transkulturelle Dimension von Becketts Werk bereits erkannt habe, bevor sie in anderen Ländern beziehungsweise in wissenschaftlichen Kontexten beachtet worden sei (Sievers 2005: 241). Für Unseld bietet sich die Radikalisierung von Exterritorialität als

8 Die Archivmaterialien im Siegfried Unseld Archiv lassen auch interessante Rückschlüsse auf Vermarktungsstrategien anderer international agierender Verleger zu. So hatte zum Beispiel Becketts US-amerikanischer Verleger Prentice (Chatto \& Windus) Beckett in den für den Autor noch sehr prekären 1930er Jahren geraten, nicht wie Joyce zu schreiben beziehungsweise sich von Joyces Einfluss zu lösen. Gleichzeitig wurde Beckett vom gleichen Verlag als Joyce-Schüler ,verkauft', um seine Anschlussfähigkeit an die moderne Weltliteratur zu gewährleisten (vgl. Nixon 2011: 3). 
Anderssprachigkeit auf einer literarischen Ebene über eine exophone Materialisierung an, indem er die Besonderheit des Literarischen gegenüber allen anderen Diskursen zum Ausgangspunkt seiner verlegerischen Tätigkeit macht. Unseld schreibt an Beckett am 1. Juni 1962:

\begin{abstract}
Autant que je sache, vous êtes le seul écrivain important qui ait écrit en deux langues. Ce fait me paraît digne d'être respecté et souligné dans une édition de vos œuvres dramatiques. J'aimerais donc publier une édition en trois langues. [...] Que pensez-vous de ce projet? Du projet d'une édition de vos œuvres dramatiques ainsi que du projet 'triglotte'? Je ne vois pas encore si la troisième solution sera réalisable, mais avant toute autre recherche j'aimerais savoir votre avis? (Brief von S. Unseld an S. Beckett vom 01.06.1962, SUA) ${ }^{9}$
\end{abstract}

In der Tat war Suhrkamp in dieser Phase der einzige Verlag, der die Zweisprachigkeit von Beckett bewusst abbildete: 1963/64 erschien das von Unseld vorgeschlagene, dreisprachige Werk in zwei Bänden als Dramatische Dichtungen, wodurch Beckett sich außerordentlich wertgeschätzt fühlte (vgl. Hartel 2011: 133f.). Unseld setzte sich stark für Beckett ein, wenngleich dieser sehr schwer zu vermarkten war, da er weder Interviews gab noch vor Publikum las (Sievers 2005: 226). Er bemühte sich ehrgeizig um seinen Autor und dessen internationale Rezeption: Nachdem er sich die Version des Theaterstücks Glückliche Tage (Happy Days) hatte zukommen lassen, die der Fischer Verlag bei der Deutschen Grammophon Gesellschaft produzieren lassen wollte, informierte er Beckett darüber, dass die Texte gekürzt waren. Beckett reagierte, indem er dem Suhrkamp Verlag die Rechte an dem Stück übertrug sowie die Rechte an allen weiteren Theaterstücken des Jahres 1962 (vgl. Hartel 2011: 135). 1969 wurde Beckett der Literaturnobelpreis zugesprochen. Das erste von Suhrkamp 1971 produzierte Taschenbuch war Becketts zentrales Stück Warten auf Godot, das ebenfalls als dreisprachige Ausgabe erschien. Die 15.000 Exemplare waren innerhalb eines Jahres vergriffen (ebd.). Unseld ermutigte Beckett auch, sein Stück Nicht ich (Not I) zu verfilmen und dabei selbst Regie zu führen.

Der Umgang mit Becketts Werk zeugt beispielhaft von der herausragenden Bedeutung Unselds für einige seiner fremdsprachigen Autor/innen. Unseld war sich seiner Stellung als gatekeeper im Sinne von Marling (2016) und seinem Anteil am internationalen Erfolg seiner Autor/innen im Allgemeinen und Becketts im

9 Für die freundliche Genehmigung, die Zitate aus den Beständen des Siegfried Unseld Archivs abzudrucken, danke ich den Urhebern bzw. deren Erben und dem Deutschen Literaturarchiv Marbach sehr herzlich. 
Speziellen sehr bewusst, wie der folgende Auszug aus einem weiteren Brief des Verlegers an den Autor nach der Nobelpreisverleihung 1969 zeigt: „Lieber Sam, ich habe Ihnen nach der Nachricht nicht geschrieben, um die Lawine von mir aus nicht zu vergrößern. Sie wissen, daß ich mich über diese Nachricht sehr freue und Sie wissen auch, daß ich Einiges zu der ganzen Sache beigetragen habe“ (Brief von S. Unseld an S. Beckett, 06.11.1969, SUA). Beckett wiederum bestätigt diese bedeutsame Rolle seines deutschen Verlegers ganz explizit in seiner Antwort (Brief von S. Beckett an S. Unseld, 06.11.1969, SUA). Der herzliche Ton der Korrespondenz zeugt dabei von der freundschaftlichen Verbundenheit von Autor und Verleger. Es war das besondere Engagement Unselds, das auch für eine Reihe lateinamerikanischer Autor/innen sehr wertvoll werden sollte. Nicht umsonst schrieb Octavio Paz in einem Brief vom 31. Juli 1980, bezugnehmend auf seine erste Deutschlandreise im Juni desselben Jahres: „Aber unsere große Entdeckung in Frankfurt war Siegfried Unseld“ (zit. nach Bürger 2010: 13). Die Publikationsgeschichte von Beckett bei Suhrkamp weist ein Bündel von Merkmalen auf, die auch für die Betrachtung der verlegerischen Selektion lateinamerikanischer Literaturen im Verlag von Bedeutung sind. Wie im Fall von Octavio Paz, um den es im Kapitel III.2.5 gehen wird, unterhielt Unseld mit Beckett eine sehr persönliche, ja: emotionale Beziehung und einen intensiven intellektuellen Austausch - wenngleich es anders als bei Paz in Becketts Fall ungleich schwieriger war, ihn mit anderen Suhrkamp-Autor/innen zusammenzubringen (Rathjen 2013: 109).

Zusammenfassend lässt sich feststellen: Auch wenn die Erfolge eines Samuel Beckett oder auch einer Autorin wie Marguerite Duras früher einsetzten, hat weder die französische noch irgendeine andere internationale Literatur bei Suhrkamp solch eine durchschlagende Rezeptionsgeschichte geschrieben wie die lateinamerikanische. Der Erwartungshorizont der Exterritorialität verabschiedet sich vom sprachphilosophischen Intellektualitätsdruck eines Samuel Beckett und wird abgelöst von einem konkreten Ort namens Macondo. Woran mag das liegen? 


\section{III.2.3. Das Lateinamerika-Programm I: die Phase des Erfolgs 1969-2000. Zwischen Universalismus und Exotismus}

Das Lateinamerika-Programm ${ }^{10}$ des Suhrkamp Verlags hatte sich im Zuge des internationalen Booms lateinamerikanischer Literatur (vgl. Kap. II.1) ab Ende der 1960er Jahre konstant entwickelt. Seit der ersten Vallejo-Ausgabe im Jahre 1963 wurde es beständig ausgeweitet - Höhepunkte sind 17 herausgebrachte Titel allein im Jahr 1976, als Lateinamerika Schwerpunktthema der Frankfurter Buchmesse war, sowie mehrere Millionen verkaufter Exemplare von Isabel Allendes Geisterhaus in den 1980er Jahren. ${ }^{11}$ Wie wird diese Literatur nun in Deutschland rezipiert? ${ }^{12}$ Wirft man einen kursorischen Blick auf Kritiken, die in der Folge der Publikationen von Hundert Jahre Einsamkeit (1967) von Gabriel García Márquez oder Das grüne Haus (1965) von Mario Vargas Llosa erschienen, so zeigt sich schnell, dass die implizite Messlatte der Begriff der Weltliteratur ist. Vittoria Borsò hat pointiert den Zusammenhang zwischen Auswahlkriterium Weltliteratur und verlegerischer Rezeption herausgearbeitet. Was sie für den bei Kiepenheuer \& Witsch herausgebrachten Roman Hundert Jahre Einsamkeit konstatiert, lässt sich auch auf Suhrkamp-Autor/innen wie Vargas Llosa übertragen: Zusammengefasst betonte die Literaturkritik, für die Zugehörigkeit zum weltliterarischen Kanon spreche die Assimilation europäischer und internationaler Traditionen und zugleich die Integration oraler Erzählstoffe aus den präkolumbischen Kulturen. Die Autor/innen hätten die europäischen und westlichen Traditionen mit den üppigen Farben der tropischen Welt angereichert. Das Paradigma Identität, im konkreten Fall die Suche nach einer spezifisch lateinamerikanischen Identität, war ein entscheidendes Kriterium, die Romane weltliterarisch vertretbar zu machen (Borsò 2004: 236).

Als ein weiteres Merkmal, das die Romane, und insbesondere La casa verde, in den Rang von Weltliteratur erhob, kann nach Borsò die Tatsache gelten, dass sich im mythischen Amazonasort am Río Marañón (vergleichbar mit dem

10 Offiziell heißt die Programmsparte bei Suhrkamp „Literatur aus Lateinamerika, Spanien und Portugal“, umfasst also auch Werke von der Iberischen Halbinsel. Der Schwerpunkt liegt aber klar bei den lateinamerikanischen Literaturen, so bezieht sich etwa auch der Text auf der Homepage des Suhrkamp Verlags, der diese Sparte vorstellt, nur auf den Subkontinent (https://www. suhrkamp.de/themen/literatur_aus_lateinamerika_spanien_und_portugal_192.html). Da der Fokus dieser Studie ebenfalls auf der lateinamerikanischen Literatur liegt, spreche ich von ,Lateinamerika-Programm:

11 Bis 2002 waren es bereits über drei Millionen (vgl. Heine 2002: 120).

12 U.a. auch dieser Phase von Suhrkamps Lateinamerika-Programm widme ich mich ebenfalls in Müller (2014a, 2015a, 2015b). 
Macondo von García Márquez) nicht nur die Geschichte Lateinamerikas, sondern auch die Geschichte der Welt ereigne. Letztere sei durch die reichen Anspielungen auf den Text der Genesis und auf archetypische Ursprungsmythen repräsentiert (ebd.). Man kann also zusammenfassend festhalten, dass die Rezeption in Literaturkritik und Literaturwissenschaft vor allem die Anschlussfähigkeit an die moderne Weltliteratur betont hat, wobei die Kombination möglichst exotistischer Alteritätsdarstellungen mit europäisch etablierten postmodernen Schreibverfahren hervorgehoben wurde.

Wie sehen im Vergleich dazu die Stellungnahmen der Verlagsindustrie aus? Hier liefern die Archivbestände des Suhrkamp Verlags, allen voran die Korrespondenzen Siegfried Unselds mit den Autor/innen, aber auch mit seinen engsten Lateinamerika-Berater/innen Michi Strausfeld und Wolfgang Eitel, anschauliches Material. Auch Begegnungen mit tonangebenden Lateinamerikanisten wie Klaus Meyer-Minnemann, Dieter Janik und Karsten Garscha finden ihren Widerhall. Im Mai 1979 schreibt Unseld in sein Notizbuch: „Octavio Paz hinterließ mir einen großen Eindruck. Eine Persönlichkeit, in der sich Poesie und Gelehrsamkeit, Weisheit und Wissenschaft vereinen. Ich habe Paz und auch Carpentier zu einem Besuch nach Deutschland eingeladen“ (Reisebericht Unseld, Paris, 20.-22.05.1979, SUA). Eines war klar: Wer zu dem illustren Kreis nicht-deutscher Suhrkamp-Autor/innen gehörte, musste als ,weltliterarisch verhandelbar' gelten. Die Gleichsetzung von Verlagsprogramm und Weltliteratur scheint unhinterfragbar. Dass diese Funktion von Octavio Paz besonders gut erfüllt wurde, mag auch mit dessen affirmativem Verständnis einer genuin europäischen Moderne zusammenhängen.

Als Maßstab für Unselds Auswahl wurden besonders gern die deutschen Klassiker herangezogen. So notierte Unseld nach einem Treffen mit Alejo Carpentier in Paris: „[Carpentier] sprach von seinem Werk und von der Welt gleichermaßen souverän. Ein Thomas Mann Lateinamerikas“ (Reisebericht Unseld, Paris, 20.-22.05.1979, SUA). Dass das Gütesiegel ,Thomas Mann‘ für Unseld als Kriterium gilt, bestätigt im Gegenzug der kubanische Autor über seine Begeisterung für germanische Literaturen. So heißt es weiter in dem Reisebericht Unselds: „Das gab nun Carpentier die Veranlassung, ausführlich über die isländischen Sagen, über die keltischen Sagen, über die deutschen Heldensagen zu sprechen und völlig verblüfft war ich dann, als er Wolfram von Eschenbachs ,Parzival‘ als wichtiges Dokument hervorhob“ (Reisebericht Unseld, Paris, 20.-22.05.1979, SUA). Ist es an dieser Stelle ein Klassiker der deutschen Moderne wie Thomas Mann, der als Vergleichsgröße dient, so zieht Unseld an anderer Stelle Verbindungen zu zeitgenössischen linksgerichteten Kulturtheoretikern wie Herbert Marcuse: 
Octavio Paz [...]. [M]an hat den Eindruck, in ihm seien Erfahrungen langer Zeiten von sozialen und literarischen Revolutionen zusammengeflossen. Für mich ist er in unserer Zeit, wo der Fortschrittsglaube und der Glaube an den historischen Prozeß zu verdämmern scheint, eine wichtige Persönlichkeit. [...] Er variiert für sich das, was Marcuse in den Römerberggesprächen zum Thema „Lebenslust“ ausgeführt hatte. (Reisebericht Unseld, Paris, 20.-22.05.1979, SUA)

\section{III.2.4. Der Suhrkamp Verlag als linksgerichtete Theorieschmiede - das Beispiel Darcy Ribeiro}

Für die deutsche Rezeption lateinamerikanischer Boom-Autoren spielt, wie auch für die weltweite Rezeption, eine politische Komponente eine wesentliche Rolle. Für diese steht das Lateinamerika-Programm des Suhrkamp Verlags nicht nur beispielhaft, sondern sie wurde vom Verlag entscheidend mitgeprägt, gilt doch der Suhrkamp Verlag als die Theorieschmiede der nichtdogmatischen Linken in Deutschland von den 1960er bis in die 1980er Jahre. ${ }^{13}$ Das gesellschaftspolitische Engagement des Verlages im Allgemeinen und auch Unselds im Besonderen, und die Rolle, die dabei die Rezeption der Boom-Literatur einnimmt, steht in direktem Zusammenhang mit dem politischen Interesse an Lateinamerika: Der Subkontinent wird in den 1960er und 1970er Jahren gewissermaßen zu einem gesellschaftspolitischen Labor, zu einem Hoffnungsträger der westeuropäischen, aber besonders der deutschen Linken, und dient dann später mit den Militärdiktaturen zur Legitimation der Kapitalismuskritik (im Sinne der Dependenztheorie). Das literarische Interesse an Lateinamerika wäre folglich als Kehrseite des politischen Interesses und des politischen Engagements für eine sozial gerechtere Welt zu lesen, sowie als fiktionale Unterstützung der theoretischen Kapitalismuskritik.

Hier scheint eine entscheidende Dimension deutscher Lateinamerika-Rezeption durch, die sich im Fall eines brasilianischen Suhrkamp-Autors zuspitzt, der zwar kein klassischer Bestseller ist und auch nicht zum Boom gehört, der aber gerade deshalb symptomatisch ist für die dominanten Rezeptionsmuster im Hinblick auf lateinamerikanische Literaturen: Darcy Ribeiro. In seinen Reisebericht zu Paris vom 20. bis 22. Mai 1979 schreibt Unseld: „Wie steht es mit dem Komplex Darcy Ribeiro? Geht das im wissenschaftlichen Bereich weiter? Ich bitte Herrn Herborth um Nachricht. Darcy Ribeiro hat einige Tage vorher eine blendende Rede zur Entgegennahme seines Ehrendoktors an der Sorbonne gehalten“(SUA).

Unselds implizite Bewertungskategorien literarischer Qualität speisen sich also auch aus einer solchen akademischen Würdigung durch eine der ältesten und

13 Vgl. dazu auch, unter etwas anderer Perspektive, Müller (2014c, 2017b). 
reputationsträchtigsten Universitäten in Old Europe. Der brasilianische Soziologe, Kulturwissenschaftler und Schriftsteller veröffentlichte 1985 Amerika und die Zivilisation bei Suhrkamp, den zweiten Band einer Serie kulturanthropologischer Studien, in denen Darcy Ribeiro die Entstehungsgeschichte und die Entwicklungsperspektiven der amerikanischen Völker auf der Folie seiner spezifischen Zivilisationstheorie untersucht. Er gehört in der deutschen Rezeption zu jener Generation lateinamerikanischer Intellektueller, die, so die Kritik, die Modernität dieses Subkontinents im Rückgriff auf die Wurzeln - die indigenen, afrikanischen und europäischen Ursprünge - begründet haben. Speziell die Romane Ribeiros wie Maira oder Mulo wurden in den Rang von Weltliteratur erhoben aufgrund der bereits verhandelten exotistischen und universalistischen Auswahlkriterien, zu denen sich bei Ribeiro noch eine weitere, ebenso entscheidende Dimension gesellte: die politische.

\section{III.2.5. Octavio Paz - ,Modell‘ eines lateinamerikanischen Weltliteraten?}

Um weiter herauszuarbeiten, welche Maßstäbe der Verleger Unseld hinsichtlich weltliterarischer Relevanz und Verhandelbarkeit lateinamerikanischer Autor/innen anlegte, lohnt es sich, im Folgenden einmal genauer auf das Beispiel Octavio Paz einzugehen. ${ }^{14}$ Am 30. April 1998, zehn Tage nach dem Tod von Octavio Paz am 20. April, schreibt Siegfried Unseld in seinem Kondolenzbrief an dessen Ehefrau Marie-José folgende Zeilen, die die zwei großen Dimensionen der Beziehung Paz/Unseld markant zu beschreiben vermögen:

For all of us Octavio is the [sic] great author. We are proud to have taken care of his work. And you can be sure that we will continue to work for him and his work, to have his books in print and to publish new ones, so that his ideas can continue to irradiate and illuminate the German public. (Brief von S. Unseld an M.-J. Paz, Frankfurt am Main, 30.04.1998, SUA)

In Doppelstimme spricht hier zum einen Unseld als findiger, erfolgreicher Geschäftsmann, der in weiser Voraussicht und mit merkantilem Fingerspitzengfühl Paz verlagsstrategisch Jahre zuvor in sein Lateinamerika-Programm aufgenommen hatte und der durch die Vergabe u.a. des Premio Cervantes (1981) sowie des Nobelpreises (1990) an Paz für sein Vertrauen in den mexikanischen Autor rückwirkend bestätigt und entlohnt wurde. Zum anderen lässt sich jedoch ebenso

14 Die folgenden Überlegungen zu Siegfried Unselds Rezeption von Octavio Paz finden sich in ähnlicher Form wieder in einem Artikel, den ich zusammen mit Sylvester Bubel verfasst habe (siehe Müller/Bubel 2016). 
Unselds Bild von - oder stärker gesagt: Projektion auf - den Literaten Paz ablesen, als der lateinamerikanische Aufklärer des 20. Jahrhunderts, dessen Ideen, ob ihrer (damaligen) Progressivität, ihrer Weitsicht, ihres Erziehungscharakters und, nicht zuletzt, ob ihres weltliterarischen Niveaus nicht versiegen dürfen. Das Paz-Bild der 1980er Jahre wird anschaulich in einem Wort des ehemaligen Bundespräsidenten Richard von Weizsäcker, der 1984 die Laudatio auf den Gewinner des Friedenspreises des Deutschen Buchhandels hielt: „Octavio Paz ist auf seinem Weg zur prägenden Stimme lateinamerikanischer Kultur geworden, zu ihrem Gewissen“ (zit. nach Suhrkamp Verlagsgeschichte 1990: 122).

Unselds programmatisches Verständnis eines verlegungswürdigen und potentiell erfolgreichen Suhrkamp-Autors im Lateinamerika-Programm ist besonders vor der Folie klassisch-etablierter - und besonders deutschsprachiger Autorenpoetiken des Suhrkamp Verlages sowie vor dem bundesrepublikanischen Bildungsbürgertums-Milieu der 1970er und 80er Jahre zu verstehen, wie sich bei genauerer Betrachtung der poetologischen, existentialistisch-religiösen und sozial-politischen Vorstellungen und Überzeugungen von Octavio Paz zeigt, die ihn für Unseld und das Suhrkamp'sche Lateinamerika-Programm interessant und relevant machten. Es handelt sich dabei um werkpoetologische Parameter, die sich als Passepartout in die von Unseld und Suhrkamp vorgegebenen weltliterarischen Leitlinien des Lateinamerika-Programms für den deutschsprachigen Markt eingliedern und funktionalisieren lassen konnten. Diesen Suhrkamp'schen Weltliteraturkriterien entsprach das Werk von Octavio Paz nach Unseld auf modellhafte Weise.

In dem Bericht seines (wahrscheinlich ersten) Treffens mit Paz, zwischen dem 20. und 22. Mai 1979 in Paris, sah sich Unseld von dem Auftreten und Kenntnisreichtum des mexikanischen Autors tief beeindruckt. Er notierte: „Eine imponierende Erscheinung, Poet und Wissenschaftler, Weiser und Wissender“ (Reisebericht Unseld, Paris, 20.-22.05.1979, SUA). Woher kommt nun diese Einschätzung Unselds hinsichtlich des Literaten sowie Intellektuellen Paz? Es drängt sich die Frage auf, ob und inwiefern Paz' Oeuvre diese (projektionistische) Sichtweise erlaubt, oder ob Unseld die Suhrkamp'schen Verlagskategorien der Person Paz und dessen Schaffen aus pragmatischen - eben verlagspolitischen - Gründen regelrecht überstülpt.

Mit welchen inhaltlichen wie formalen Aspekten und Mitteln vermag nun also Paz, der „Weise und Wissende“, seinen Status als lateinamerikanischer Polyhistor und Aufklärer in der Wahrnehmung Unselds zu zementieren? Um diesen Konnex besser zu verstehen, bedarf es einer kurzen und fragmentarischen Begriffsbeschreibung des Unseld'schen Verständnisses eines ,Aufklärers“ beziehungsweise ,Wissenden' von Weltrang; dieses lässt sich besonders implizit an den Vergleichskategorien festmachen, mit denen Unseld Paz für sein eigenes Kunstverständnis fassbar $\mathrm{zu}$ machen versuchte. So sieht Unseld die Trias Cortázar - Paz - Carpentier als die „Großen lateinamerikanischer Literatur. Alle 
drei beeindruckend, weil sie nicht nur das Eigene, den eigenen Kontinent, sondern auch die anderen in sich reflektieren" (Reisebericht Unseld, Paris, 20.-22.05.1979, SUA). Unseld kategorisiert Paz also als einen ,universalistischen“ Autor, der sich jenseits der eigenen nationalen und ethnischen Be- und Eingrenzungen mit den - vermeintlich - höheren, existentiellen Fragen des Daseins in seinem Werk auseinanderzusetzten vermag. Ein „Weltenreisender“15, der gedanklich zwischen den Referenzpunkten Lateinamerika und Europa oszilliert.

Hinter dieser intellektuellen Adelung von Paz zum Grandseigneur der lateinamerikanischen Literatur - neben Julio Cortázar und Alejo Carpentier, dem „Thomas Mann Lateinamerikas“ (Reisebericht Unseld, Paris, 20.-22.05.1979, SUA) - ließe sich aber auch ein verlagspolitischer Kniff vermuten: Mit Kuba, Mexiko und Argentinien konnte Unseld so für das deutsche Nachkriegsbürgertum die politisch interessantesten Regionen Lateinamerikas mit einem Gesicht versehen und in seinem Programm greifbar machen.

Neben verlagspolitischen Erwägungen und dem angesprochenen Aufklärungs-Schema umfasst die Unseld'sche Rezeption von Paz' Poetik indes noch einen dritten, entscheidenden Aspekt: Der Verleger ist zutiefst angetan von einem Vortrag von Paz in Frankfurt am Main im Sommer 1980 und extrahiert aus den ästhetischen und poetologischen Erwägungen des Mexikaners die für ihn zentrale Aussage: „Ja, es ist wahr: ,America Latina es una cultura“ - ,Lateinamerika ist eine Kultur““ (Brief von S. Unseld an O. Paz, 04.07.1980, SUA). Trotz der so hohen Diversität hinsichtlich Politik, Kultur, Ethnie, Sprache und letztendlich der Literatur, möchte Unseld Lateinamerika als ein homogenes Ganzes verstanden wissen, dessen politischer Individuationsprozess von der zeitgenössischen Literatur nicht nur reflektierend begleitet, sondern aktiv angeleitet und vorangetrieben wird. Und so wird lateinamerikanische Literatur von Suhrkamp oftmals mit der Vokabel ,existentialistisch` beworben und Lateinamerika als eine Art ,unbefleckter` emotionaler (Kontinental-)Raum in der Konstitutionsphase. Es verwundert also nicht, dass das Lateinamerika-Programm des Suhrkamp Verlags im Jahr 1979 unter dem Titel 17 Autoren schreiben am Roman des lateinamerikanischen Kontinents publiziert wurde und Unseld darin griffig kommentiert:

Von allen außerdeutschen zeitgenössischen Literaturen scheint mir die lateinamerikanische sicherlich für das nächste Jahrzehnt die wichtigste zu sein. Es ist eine besondere Art existentieller Literatur, wie sie in anderen Ländern, aus welchen Gründen auch immer, nur noch in einzelnen Fällen geschrieben werden kann. Diese Literatur macht Mut zu einer neuen Erziehung zu Gefühlen und ihre so realen Gestalten scheuen sich nicht, Werte des Lebens zu benennen und in ihrem Rang zu bestimmen. (SUA)

15 So Michi Strausfeld in einem verlagsinternen Entwurf, der mit „es-NF-Lateinamerika“ überschrieben ist und vermutlich aus dem Jahr 1980 stammt (Quelle: SUA). 
Eine solche „existentielle“ Literatur Lateinamerikas, die die „Werte des Lebens zu benennen“ und gar eine neue éducation sentimentale anzufachen vermag, folgt in Unselds Verständnis jedoch bekannten Koordinaten: Über das Gedicht „Piedra de Sol“16, von dessen - wie er es ausdrückt - „Hunger nach Sein“ (Reisebericht Unseld, Paris, 20.-22.05.1979, SUA) er explizit beeindruckt war, schreibt Unseld, dass ihn besonders die Art und Weise, wie das Gedicht endet, faszinierte, das „Symbol des Flusses, ,als der, der sich windet, verdrängt, zurückweicht, einen Umweg wandelt und immer ankommt‘, das weiche Wasser in Bewegung“ (ebd.). Es scheint kein Zufall $\mathrm{zu}$ sein, dass Unseld gerade solche in Literatur- und Philosophiegeschichte topisch besetzten Metaphern, Bilder und Tropen wie das heraklitische Panta rhei in Paz' Werk wertzuschätzen wusste. Bedenkt man die Ausrichtung des Lateinamerika-Programms und ihre Strategie der ,lateinamerikanischen Erweckung;, bedarf es solcher universalistisch auslegbarer und geschichtsschwangerer Metaphoriken, die es vermögen, Lateinamerika dem nahezu unwissenden deutschsprachigen Publikum griffig näherzubringen.

Obendrein qualifizierten sie den mexikanischen - und damit für die damalige Zeit im deutschsprachigen Raum noch exotisch anmutenden - Autor Paz, in die Gruppe historisch bedeutender Suhrkamp-Autor/innen eingemeindet zu werden. Unselds Bestreben war es, in seiner heute historisch gewordenen Bibliothek der Klassiker der Moderne „keine Ausgrabungen abseitiger Gelegenheitsarbeiten [zu publizieren], vielmehr trotz divergierendster Themen eine Geschlossenheit im Ziel [anzustreben], die die Behauptung rechtfertigt: Hier wird Literatur zur Flucht in das Leben“ (Unseld 1989). Diese Flucht in das Leben scheint in Unselds Verständnis etwas spezifisch Modernistisches zu sein, geprägt von Friedrich Nietzsches emphatischem ,Ja zum Leben“ und Bergsons élan vital oder auch der engagierten Literatur von der Weimarer Republik (mit Hesse und Brecht) bis zu ihren existentialistischen Ausprägungen (mit Sartre und Camus); und je mehr Überschneidungspunkte mit diesen Größen sich in den Ästhetiken der Lateinamerikaner/innen finden lassen, desto wertvoller erscheint Unseld ihr Werk.

Dass für Unseld Literatur mit gesellschaftlichem Engagement und sozialpolitischem Handeln untrennbar zusammenhängt, zeigt nicht nur die programmatische Ausrichtung der Bibliothek Suhrkamp nach dem Zweiten Weltkrieg, sondern auch seine zuhöchst positive Bewertung gesellschaftlich engagierter Literat/innen, die eben nicht im Elfenbeinturm des l'art pour l'art verweilen (wie etwa Darcy Ribeiro). Paz' Ästhetik grenzt sich von solchen künstlerischen Eskapismen

16 Bei dem Gedicht handelt es sich um eines von Octavio Paz’ Hauptwerken, das Unseld nachweislich gelesen hat. Veröffentlicht wurde es auf Deutsch bei Suhrkamp 1977 in dem Band Octavio Paz. Gedichte und 1979 in Suche nach einer Mitte. Die großen Gedichte. 
(wie wir sie aus dem Symbolismus kennen) ab, ohne sich freilich zum literarischen Sprachrohr politischer und gesellschaftlicher Positionen funktionalisieren zu lassen. Politisch engagierte Literatur vermag sich allzu häufig nicht von den teleologischen Denkmustern bürgerlicher Rationalität zu lösen, sodass es gerade als Aufgabe des Dichters angesehen werden kann, durch Poesie einen geschützten Raum zu schaffen, in dem supratemporale, vom Zeitgeist unbeeinflusste Ideen und Episteme überleben können - befreit von jeglicher Vereinnahmung durch bürgerliche Denkkategorien. So schreibt Paz etwa in Sor Juana Inés de la Cruz o Las trampas de la fe:

El racionalismo burgués es, por decirlo así, constitucionalmente adverso a la poesía. De ahí que a poesía, desde los orígenes de la era moderna - o sea: desde las postrimerías del siglo XVIII - se haya manifestado como rebelión. La poesía no es género moderno; su naturaleza profunda es hostil o indiferente a los dogmas de la modernidad: el progreso y la sobrevaloración del futuro [...]. La poesía, cualquiera que sea el contenido manifiesto del poema, es siempre una transgresión de la racionalidad y la moralidad de la sociedad burguesa. Nuestra sociedad cree en la historia - periódico, radio, televisión: el ahora - y la poesía es, por naturaleza, extemporánea. (Paz 1985 [1982]: 16)

Paz verwehrt sich hier gegen Politisierung, Vereinnahmung und Einflussnahme bürgerlicher Wertkategorien auf literarische Produktion und weist der Poesie und dem Poeten eine einzigartige rebellische Kraft $\mathrm{zu}$, die eine genuin andere Epistemologie zu produzieren vermag: nämlich ein überzeitliches Lebenswissen, das Moralität generiert, jenseits von Positivismus, Rationalismus und Empirismus. Durch diese eigene Art der literarischen Politisierung wird der Dichter seiner Verantwortung gegenüber der Gesellschaft gerecht, die Paz in „La letra y el cetro“ betont: „Pero no podemos renegar de la política; sería peor que escupir contra el cielo: escupir contra nosotros mismos“ (Paz 2002 [1972]: 754f.).

Jene schöpferische, rebellische Kraft, die Paz in dem Medium der Literatur und vor allem der Poesie sieht, ist es auch, die Unseld am Werk des Mexikaners so schätzt. Poesie als eigenständige epistemologische Kategorie zu etablieren, dieser Versuch findet sich bei vielen modernistischen Dichter/innen im Umkreis des Suhrkamp Verlages, so bei dem von Unseld so hochgeschätzten Hugo von Hofmannsthal, dessen Essay Der Dichter und diese Zeit aus dem Jahr $1906^{17}$ frappierende Ähnlichkeiten zu den Gedanken Paz’ aufweist.

17 Es handelt sich um einen Vortrag, den Hugo von Hofmannsthal Ende 1906 mehrfach hielt. Erstmals abgedruckt wurde „Der Dichter und diese Zeit“ im März 1907 in der Neuen Rundschau in Berlin. 
Keinen Gedanken der sich an ihn [den Dichter] drängt, darf er von sich scheuchen, als sei er aus einer anderen Ordnung der Dinge. Denn in seine Ordnung der Dinge muß jedes Ding hineinpassen. In ihm muß und will alles zusammenkommen. Er ist es, der in sich die Elemente der Zeit verknüpft. In ihm oder nirgends ist Gegenwart. [...] Wie der innerste Sinn aller Menschen Zeit und Raum und die Welt der Dinge um sie her schafft, so schafft er aus Vergangenheit und Gegenwart, aus Tier und Mensch und Traum und Ding, aus Groß und Klein, aus Erhabenem und Nichtigem, die Welt der Bezüge. (Hofmannsthal 1979 [1906]: 67f.)

Hofmannsthal stellte für Unseld gewiss eines der wichtigsten europäischen Tertia Comparationis dar, um Paz' Oeuvre evaluieren zu können. So zeugt ein Brief Unselds an Paz vom 4. Juli 1980, in dem Unseld einen einwöchigen Aufenthalt des Schriftstellers und dessen Ehefrau Marie-José in Deutschland Revue passieren lässt, von dem Drang des Verlegers, Paz in die Tradition etablierter SuhrkampAutor/innen und deutschsprachiger Geistesgrößen zu stellen. Während der Reise durch Westdeutschland (Bonn, Frankfurt, Heidelberg) ist nicht nur ein Besuch „im Goethe-Haus selbstverständlich“, nein, man lässt sich auch in der GoetheBibliothek „Hofmannsthal-Schätze“ zeigen, denn Hofmannsthal, so Unseld, „war ja einer der wenigen deutschen Dichter, die des Spanischen mächtig waren“ (Brief von S. Unseld an O. Paz, 04.07.1980, SUA). Vor der Hofmannsthal'schen Vergleichsfolie gewinnt folgendes Zitat Unselds aus dem bereits zitierten Reisebericht von 1979 an Bedeutung:

Für mich ist er [0. Paz] in unserer Zeit, wo der Fortschrittsglaube und Glaube an den historischen Prozess zu verdämmern scheint, eine wichtige Persönlichkeit. Seine Erfahrung aus einem halben Jahrhundert der Umstürze: niemand weiß, wie die Zukunft aussieht, wir müssen wachsam sein und aufpassen, skeptisch sein, aber wir sollten doch auch hoffen. Und für ihn ist das Wichtigste: der höchste Wert ist nicht die Zukunft, sondern die Gegenwart. „Die Zukunft ist nicht die Zeit der Liebe: was der Mensch in Wahrheit will, das will er jetzt. Derjenige, der das Haus der künftigen Glückseligkeit konstruiert, errichtet das Gefängnis der Gegenwart.“ Und das Urteil über den Fortschritt lautet: „Er hat die Geschichte mit den Wundern und Monstren der Technik bevölkert, aber er hat das Leben der Menschen entvölkert, er hat uns mehr Dinge gegeben, nicht mehr Sein.“ (Reisebericht Unseld, Paris, 20.-22.05.1979, SUA)

Auch hier kritisiert Unseld den bürgerlichen Fortschrittsglauben und das dichotome Verhältnis zwischen - mit Erich Fromm gesprochen - „Sein und Haben“ bürgerlicher Wertkategorien. Paz drückt diese Ambivalenz in einem 1996 erschienenen Interviewband folgendermaßen aus:

hay una oposición radical entre los valores de la sociedad moderna y la poesía. La cultura de la sociedad capitalista está basada fundamentalmente en la moral de la utilidad. Y la poesía siempre es un gasto, un desperdicio. Hay incompatibilidad entre la moral burguesa - que es la moral del ahorro - y la moral poética, que es la moral del dar, del despilfarro. (Peralta 2014 [1996]: 40) 
Hier kann man Anklänge an den radikalen Sartre'schen Existentialismus der 1950er Jahre erkennen und noch einmal den Versuch Unselds, Paz in sein Kaleidoskop einflussreicher (europäischer) Denker/innen und Autor/innen einzugruppieren.

Auffallend ist Unselds Bemühen um Legitimation: Er möchte die lateinamerikanische Literatur so ernst genommen wissen, dass sie berechtigte Aufnahme in den Kanon der Weltliteratur finden kann: „Ich muß hinzufügen, daß die lateinamerikanische Literatur ein Neuankömmling ist. Sie ist die jüngste aller westlichen Literaturen. [...] Lateinamerika ist ein Ferner Westen“ (Reisebericht Unseld, Paris, 20.-22.05.1979, SUA). Mit dem Etikett ,Ferner Westen“ werden die etablierte Kategorie eines affirmativen Orientalismus - ebenfalls ein weltliterarisches Indiz - in eine transatlantische Sphäre verlagert und damit gewachsene Wertmaßstäbe für die literarische Inszenierung des Anderen als des Exotischen übernommen. Die Verbindung von Anschlussfähigkeit an die deutsche Bildungstradition (in Form hochkanonischer Autor/innen) mit linksgerichteter Theoriebildung schuf einen dem Verlag dienlichen Rezeptionsfilter, der pragmatisch zentrale Themenfelder der Suhrkamp Culture verband: Religiosität, Ethik, Existentialismus. Paz formuliert in einer Vorlesung, die er am 4. Dezember 1976 an der Yale University hielt:

Nuestra literatura está hecha de las relaciones - choques, influencias, diálogos, polémicas. Monólogos entre unas cuantas personalidades y unas cuantas tendencias literarias y estilos que han cristalizado en una obra. Esas obras han traspasado las fronteras nacionales y las ideológicas. La unidad de la desunida Hispanoamérica está en su literatura. (Paz 2011 [1976])

\section{III.2.6. Isabel Allende - ein „Glücksfall für die Literatur“}

Isabel Allende passte zunächst nicht in ein solches Konzept einer zwar auch von Zusammenstößen geprägten, aber doch eine wichtige Einheit schaffenden lateinamerikanischen Literatur. In Lateinamerika hatte sie als Autorin wenig Rückhalt, viele Kritiker/innen meinten, sie bediene lediglich einen westlichen Literaturgeschmack. Allerdings hat ihr Werk das Programm des Suhrkamp Verlages sehr geprägt - wenn auch aus gänzlich anderen Gründen als das eines Octavio Paz. Über die Veröffentlichung von Isabel Allendes Erstling Das Geisterhaus (1984) schrieb Siegfried Unseld in der Suhrkamp Verlagsgeschichte 1950-1990:

Am 12. März erscheint Isabel Allendes Roman Das Geisterhaus, aus dem Spanischen übersetzt von Anneliese Botond. Es ist das erste Buch dieser Autorin, der Nichte des chilenischen Präsidenten Allende, die nach dem Putsch vom 11. September 1973 Chile verließ und nun mit 
ihrer Familie als Journalistin in Venezuela lebt. [...] Nirgendwo war der Erfolg so groß wie in Deutschland [...]. Das Buch ist viele Monate lang die Nummer 1 der Bestsellerliste des „Spiegel“. Dieser „Glücksfall für die Literatur“ ist in Deutschland (Stand Juni 1990) mit zwei Millionen Exemplaren verbreitet. (1990: 163f.) ${ }^{18}$

Isabel Allende ( ${ }^{\star}$ Lima 1942) ist heute eine der meistgelesenen Autor/innen weltweit und wohl auch die kommerziell erfolgreichste literarische Stimme der spanischsprachigen Welt. ${ }^{19}$ Nach eigenen Angaben hat sie 23 Bücher veröffentlicht, die in 42 Sprachen übersetzt wurden und von denen insgesamt 74 Millionen Exemplare verkauft wurden. ${ }^{20}$ Die rein quantitative Verbreitung ihres Werkes sei im lateinamerikanischen Kontext nur noch vergleichbar etwa mit jener eines Paulo Coelho, bemerkt Ilan Stavans 2001 im Times Literary Supplement (zit. nach Heine 2002: 116). Im Verlauf ihrer Karriere erhielt sie zahlreiche Ehrendoktorwürden und internationale Literaturpreise; mittlerweile sind es über sechzig Preise aus über 15 Ländern. ${ }^{21}$ Zwei ihrer Romane wurden verfilmt. Zum einen La casa de los espiritus als The House of the Spirits (1993) mit Meryl Streep, Jeremy Irons und Glenn Close unter der Regie von Bille August und zum anderen De amor y sombras als Of Love and Shadow (1994) mit Antonio Banderas und Jennifer Connelly unter der Regie von Betty Kaplan (Strausfeld 2012: 71).

Innerhalb dieser globalen Erfolgsgeschichte nimmt die Rezeption in Deutschland - wie Siegfried Unseld es in dem Eingangszitat andeutet - nochmals eine Sonderstellung ein, kommerziell ist Allende sicherlich ein singulärer verlegerischer „Glücksfall“. Die Absatzzahlen der im Suhrkamp Verlag erschienenen deutschen Übersetzungen, insbesondere von Das Geisterhaus, können nur als phänomenal bezeichnet werden und haben Allendes fulminante internationale Karriere nicht unerheblich befeuert. In einem Interview aus dem Jahr 2002 führt Jorge Heine nach Sprachen differenzierte Verkaufszahlen für ihre ersten neun Werke

18 Es ist sehr schwierig, belastbare Daten zu den Verkaufszahlen von Büchern zu bekommen, da Verlage als wirtschaftliche Unternehmen keine Rechenschaftspflicht gegenüber der Öffentlichkeit haben. Teilweise divergieren die Angaben erheblich.

19 Die Latin American Herald Tribune bezeichnet Allende in einem Artikel aus dem Oktober 2009 als „the world's most widely read Spanish-language author“ („Isabel Allende“ 2009).

20 Stand März 2019. Die Angaben stammen von der Internetseite der Isabel Allende Foundation, einer Stiftung der Autorin, die sich weltweit für die Rechte von Frauen und Mädchen einsetzt (https://s3-us-west-1.amazonaws.com/isabelallende.com/assets/bio/Bio_Isabel-en.pdf?kaodas5 41ks). Suhrkamp spricht von „mehr als 57 Millionen Exemplare[n]“ (https://www.suhrkamp.de/ isabel-allende_939.html) und stützt sich dabei offenbar auf die zum Zeitpunkt der Recherche mehr als sechs Jahre alten Zahlen von Strausfeld (2012: 71).

21 Auch diese Zahlen stammen von der Internetseite der Isabel Allende Foundation (https://s3us-west-1.amazonaws.com/isabelallende.com/assets/bio/Bio_Isabel-en.pdf?kaodas541ks). 
auf. $^{22}$ Daraus lässt sich ablesen, dass von den rund 32,5 Millionen weltweit zirkulierenden Exemplaren dieser Romane und Erzählungen knapp acht Millionen, also fast ein Viertel, deutsche Übersetzungen sind und also von Suhrkamp verlegt wurden. Diese Zahlen sind auch insofern beeindruckend, als sie deutlich machen, dass von diesen neun Titeln mehr deutsche Ausgaben verkauft wurden als in jeder anderen Sprache, auch mehr als auf Spanisch - in Spanien und Lateinamerika zusammen (knapp 6,6 Mio.). Besonders auffällig ist dies bei Allendes Debütroman La casa de los espíritus, der allein bis 2002 in Deutschland über drei Millionen Mal über den Ladentisch ging (bei weltweit 10,8 Millionen verkaufter Exemplare) (Heine 2002: 120). ${ }^{23}$

Wie war es dazu gekommen? Das Manuskript von La casa de los espíritus wurde zunächst von einigen lateinamerikanischen Verlagshäusern abgelehnt, weshalb die Autorin es der spanischen Agentin Carmen Balcells schickte, die - wie allgemein bekannt ist - unter anderem auch Gabriel García Márquez vertrat. Der Roman wurde schließlich 1982 vom spanischen Plaza \& Janés Verlag publiziert und gewann im selben Jahr den Premio Mazatlán (Brown 1994: 37). Bei der Buchvorstellung in Barcelona wurde Michi Strausfeld, die zum damaligen Zeitpunkt als unabhängiger Literatur-Scout für den Suhrkamp Verlag tätig war, auf die chilenische Schriftstellerin aufmerksam. Strausfeld schrieb eine Empfehlung an den Verlag, der daraufhin die deutschen Rechte erwarb und den Roman von Anneliese Botond ins Deutsche übersetzten ließ. 1984 wurde er unter dem Titel Das Geisterhaus publiziert (ebd.). Botond gewann für ihre Übersetzung den Johann-Heinrich-Voß-Preis, der von der Akademie für Sprache und Dichtung für „,hervorragende Leistungen auf dem Gebiet der Übersetzung“ verliehen wird. Das Geisterhaus schoss 1985 auf Platz 1 der deutschen Bestsellerliste und blieb

22 Heine (2002: 120) nennt Zahlen für folgende Länder beziehungsweise Sprachräume: Deutschland (es ist anzunehmen, dass der gesamte deutsche Sprachraum, also inklusive Österreich und Schweiz, gemeint ist), Brasilien, Dänemark, Spanien/Lateinamerika, Finnland, Frankreich/Kanada, Griechenland, Niederlande, Island, Israel, Italien, Japan, Norwegen, Portugal, Schweden, Türkei, USA/Großbritannien.

23 Dass sich Das Geisterhaus (in der deutschen Übersetzung) drei Millionen Mal verkauft hat, führt 2007 auch Sperschneider an (2007: 108). Bereits 1987 sprach Gottfried Honnefelder, damals Geschäftsführer bei Suhrkamp, gegenüber der Zeit von 500.000 verkauften Exemplaren (Greiner 1987). Unseld gibt dann drei Jahre später, wie bereits zitiert, die Zahl von zwei Millionen an (Suhrkamp Verlagsgeschichte 1990: 163f.). Was die weltweite Zirkulation des Romans betrifft, so ist auf der Seite des Suhrkamp Verlags die unglaubliche Zahl von 51 Millionen verkaufter Exemplare zu lesen (https://www.suhrkamp.de/isabel-allende/das-geisterhaus_942.html). Es ist anzunehmen, dass hier ein Fehler vorliegt und Suhrkamp eine alte Zahl für die Gesamtverkäufe aller Bücher Allendes, die so immer noch auf der deutschen Wikipedia-Seite der Autorin steht (https:// de.wikipedia.org/wiki/Isabel_Allende), fälschlich allein dem Romanerstling zuordnet. 
während des gesamten Jahres unter den ersten zehn (Gerling 2007: 74). Zunächst wurde Allende im Programm des Suhrkamp Verlags unter der Kategorie ,junge Autorin' vermarktet (Strausfeld 2007: 165). Es gelang ihr, auch die in kurzen Abständen folgenden nächsten drei Romane beziehungsweise Erzählbände in der deutschen Bestsellerliste zu platzieren: Von Liebe und Schatten (1986; Original: De amor y de sombra, 1984), Eva Luna (1988; Original: Eva Luna, 1987), Geschichten der Eva Luna (1990; Original: Cuentos de Eva Luna, 1989) (Brown 1994: 60). Und auch wenn die imposanten Verkaufszahlen von Das Geisterhaus bei weitem nicht wieder erreicht wurden, so finden sich die Romane von Isabel Allende bis heute zuverlässig unter den Top Ten der Spiegel-Bestsellerliste. ${ }^{24}$ Ihr enormer kommerzieller Erfolg hatte zur Folge, dass sich der zeitliche Abstand zwischen der Veröffentlichung ihrer Werke im Original und dem Erscheinen der deutschen Übersetzung $^{25}$ mehr und mehr verringerte, bis hin $\mathrm{zu}$ einer beinahe zeitgleichen Publikation, während die Zeitspanne bei weniger erfolgreichen Autor/innen wesentlich größer ist und mehrere Jahre umfassen kann (Sperschneider 2007: 108).

Welche Rezeptionsmuster sind in Bezug auf die Literatur von Isabel Allende in diesem Kontext von Bedeutung? Die lateinamerikanische Literaturkritik hat der Autorin vorgeworfen, sie bediene nur einen westlichen Literaturgeschmack, sie habe sich von Lateinamerika abgewandt und sei im Herzen US-Amerikanerin geworden, ihr sei jedes Mittel zur Vermarktung ihrer Bücher recht - speziell ihre Selbstinszenierung als politische Exilantin wird ihr übelgenommen. ${ }^{26}$ Vor diesem Hintergrund wird erst verständlich, dass der Autorin - trotz ihres großen internationalen literarischen Renommees - erst im Jahr 2010 in ihrem Heimatland Chile eine offizielle Anerkennung zuteil wurde in Form des Nationalpreises für Literatur (Strausfeld 2012: 71). Im anglophonen Raum, wo ihre Bücher ebenfalls Verkaufsschlager sind, fallen die Reaktionen auf das Phänomen Allende zwiespältig aus. Teilweise werden ihre Romane als wenig überzeugende Nachahmung der großen Klassiker des Magischen Realismus abgelehnt, so schreibt etwa 2001 Ilan Stavans im Times Literary Supplement, Allende sei die erste Frau gewesen, die im Kontext des lateinamerikanischen Boom $^{27}$ ernst genommen worden sei, aber zugleich

24 Vgl. zuletzt Ein unvergänglicher Sommer, im Sommer 2018 auf Rang 4.

25 Übersetzte noch Anneliese Botond Allendes Debütroman, so übernahm Dagmar Ploetz die Übertragung von De amor y de sombra ins Deutsche, die jedoch, anders als ihre Vorgängerin, für ihre Übersetzung stark kritisiert wurde (vgl. Brown 1994: 39). Der dritte Roman, Eva Luna, wurde dann von Lieselotte Kolanoske übersetzt, die 15 Jahre lang Allendes deutsche Stimme blieb, bis 2003 Svenja Becker diese Rolle übernahm.

26 Vgl. Heine (2002: 116), der eine Auswahl kritischer Stimmen zitiert.

27 So die Literaturkritik; aus literaturwissenschaftlicher Perspektive zählt Isabel Allende nicht zum Boom der lateinamerikanischen Literatur. 
habe sie auch das Ende dieses literarischen Experimentes eingeläutet und es durch die reine Unterhaltung ersetzt. Allende habe die Umwandlung von Literatur in ein für den breiten Geschmack ausgelegtes Konsumgut auf die Spitze getrieben (zit. nach Heine 2002: 116). Doch es gibt auch andere Stimmen: Philip Swanson etwa konstatiert mit Blick auf die Kritiken zu La casa de los espíritus eine vorherrschende Erwartungshaltung, die sich vor allem auf Politik und Komplexität konzentriere. Dabei gehe es stets um eine „radikal linke Politik, die durch radikal subversive Erzählformen ausgedrückt wird“ (Swanson 2003: 57). Dahingegen sieht Swanson die große Stärke des Romans gerade darin, dass er mit der Tendenz lateinamerikanischer Literaturen hin zum Komplexen und Unbekannten bricht und den sogenannten Magischen Realismus in eine kommunikativere Form bringt (ebd.).

Das deutschsprachige Feuilleton nimmt Das Geisterhaus und die nachfolgenden Romane insgesamt wohlwollender auf, wobei immer wieder auch Kritik an als seicht oder kommerziell wahrgenommenen Seiten von Allendes Werk formuliert wird. Achtzehn Jahre nach dem Erscheinen von Das Geisterhaus porträtiert Martin Ebel in der Frankfurter Allgemeinen Zeitung rückblickend das literarische Schaffen Allendes als eine „kommode[] und softe[] Variante lateinamerikanischer Moderne: Isabel Allende gab den weiblichen García Márquez, ohne erzählerisches Risiko, ohne Kraft, Atem und Furor des Nobelpreisträgers, ohne seine Härten und Ansprüche. Was sie bot, war sozusagen literarischer Kuschelsex“ (Ebel 2002: 40). Über Geschichten der Eva Luna heißt es an anderer Stelle: „Allzu verführerisch erschien der exotisch parfümierte Zauber einer Erzählkunst, die den nordischen Trübsinn auf so unterhaltsame Weise vertrieb“ (Brown 1994: 123). Eva Luna wird unter anderem als „exotisches Märchen“ (Brown 1994: 121) bezeichnet, welches „mit dicken Tupfen aus Schweiß, Sperma, Blut“ das „südamerikanische Ambiente koloriert“ (Brown 1994: 126). Hier zeigt sich in aller Deutlichkeit ein zentraler Rezeptionsrahmen, in dem der Fokus auf einen überbordenden, sinnlich grundierten Exotismus gerichtet wird, der offenbar für die Publikumsgunst eine massive Rolle spielte. In diese Richtung weist auch die Vermarktungsstrategie des Suhrkamp Verlags, der Das Geisterhaus zum einen als einen „der erfolgreichsten Romane der Weltliteratur“ anpreist und zum anderen mit Hilfe von Exotismus und sinnlichen Versprechen bewirbt: „In einer von Geistern bewohnten Welt, voller Geheimnisse und dunkler Ahnungen lässt Isabel Allende Figuren aus Fleisch und Blut auftreten, die von ihren Überzeugungen und Leidenschaften getrieben sind.“ ${ }^{28}$ Die Rolle, die Isabel Allendes Romanen im Lateinamerika-Programm des

28 https://www.suhrkamp.de/isabel-allende/das-geisterhaus_942.html. 
Suhrkamp Verlags zugewiesen wurde, bringt Martin Ebel in seiner Rezension von Portrait in Sepia auf den Punkt:

In der eindrucksvollen Riege internationaler Autoren des Suhrkamp Verlags ist Isabel Allende nicht gerade ein Schmuckstück - in der Jahresbilanz aber durchaus. Es gibt Kollegen, bei denen es sich umgekehrt verhält, und beides soll und darf ja in einer verlegerischen Mischkalkulation auch so sein. (Ebel 2002: 40)

\section{III.2.7. Exkurs: Nordamerikanische Literaturen bei Suhrkamp - das Beispiel William Faulkner}

Ein Blick auf William Faulkner lohnt sich hier allein schon deshalb, weil die Mehrheit der Autor/innen des lateinamerikanischen Boom von sich sagt, nicht ohne ihn schreiben zu können. Die US-amerikanischen Autor/innen, denen kein mit den lateinamerikanischen vergleichbarer Erfolg beschieden war, fanden ihren Platz vor allem in der Bibliothek Suhrkamp, die Werke der klassischen Moderne von William Faulkner, Sherwood Anderson, Gertrude Stein sowie Truman Capote vertrat. Der 2011 verstorbene, aus Martinique stammende Literat und Kulturtheoretiker Édouard Glissant widmete Faulkner mit Faulkner, Mississippi einen 300-seitigen literarischen Essay. Die starke Rezeption gerade durch lateinamerikanische Autor/innen ist nicht ohne Berücksichtigung der omnipräsenten hemisphärischen Dimension der Amerikas in Faulkners Werk zu verstehen. Auf die daraus erwachsenden kulturtheoretischen Implikationen hat Marcel Vejmelka sehr überzeugend hingewiesen: Das von Faulkner im Zuge zahlreicher Romane und Erzählungen um die Stadt Jefferson herum ausgestaltete, fiktive Yoknapatawpha County beinhaltet eine Vielzahl historischer Dimensionen, die es mit anderen Räumen des amerikanischen Kontinents verbinden. ${ }^{29}$ Die „Grundlogiken der kolonialen und postkolonialen Konfiguration“ seiner Kultur, die in Yoknapatawpha sichtbar werden, setzten Faulkners Süden der USA in komplexe Wechselbeziehungen mit anderen Regionen des von der zugleich kolonialen wie kapitalistischen Maschinerie der Plantagenwirtschaft geprägten Plantation America (Vejmelka 2009: 157f.). Die von Faulkner zur Darstellung gebrachte historische Erfahrung der Plantagenwirtschaft - der weißen Landbesitzer und afrikanischen Sklaven, sowie des vom modernen Norden der USA eroberten Deep South, des Leidens einer Region, die gewaltsam in das moderne nationale Projekt der (nördlichen) USA eingegliedert und intern kolonisiert wurde, die zugleich

29 Für die Ausführungen zu Faulkner orientiere ich mich direkt an dem wertvollen Beitrag von Marcel Vejmelka (2009: hier S. 157). 
aber um ihre Schuldhaftigkeit als Sklavengesellschaft weiß - bildet eine relationale Logik, die es ermöglicht, Yoknapatawpha County aus seiner regionalen Spezifik heraus im Hinblick auf grundlegende Fragen der kulturellen Konfiguration und Identitäten der Amerikas zu analysieren (Vejmelka 2009: 158). Faulkners Werk situiere sich somit auf der Grenze innerhalb der Amerikas, die jeweils von Norden und Süden her angenommen, behauptet, angezweifelt oder problematisiert wird (ebd.).

Es liegt nahe, dass Édouard Glissant dieses Material dankbar weiter konstruiert: In seiner reiseliterarischen Skizze mit dem Titel Faulkner, Mississippi wird in Bezug auf Faulkners Anwesen in der Nähe von Oxford - von einem Süden in den anderen Süden - die von dem Anthropologen Gilberto Freyre 1933 im brasilianischen Nordosten untersuchte Konstellation von „Herrenhaus und Sklavenhütte“ sichtbar (Vejmelka 2009: 172f.). Vejmelka arbeitet heraus, inwiefern Glissant diese „räumliche Miniatur Brasiliens“ - seiner ökonomischen, sozialen und insbesondere ethnischen Entstehung - auf die Plantagen des US-amerikanischen Südens überträgt, in denen Leben und Werk Faulkners angesiedelt sind (2009: 163). Gerade im ,zeitlichen Spagat‘ dieser hemisphärischen Amerika-Konstruktionen zwischen Faulkner und Glissant zeigt sich die Einheit der Amerikas in Schreib- und Leseakt zugleich. Diese kulturtheoretisch in der Lateinamerikanistik unterschätzte Dimension spiegelt sich auch in der deutschen Rezeption und hat damit wiederum Folgen für weltweite Kanonisierungsprozesse. Wie positioniert sich nun Suhrkamp ganz konkret?

Von Faulkner erschien wenig. Nur die drei Erzählungen Als ich im Sterben lag (1961), Der Bär (1953) und Wilde Palmen (1957) kamen bei Suhrkamp heraus; da

sie jedoch erst nach der Nobelpreisvergabe erschienen, kann sich der Verlag keine Pionierarbeit auf die Fahnen schreiben. Glissant wurde bis heute nur in dem kleinen, stark programmatisch ausgerichteten Heidelberger Verlag Das Wunderhorn publiziert. Die Nicht-Publikation kann an dieser Stelle nur die Frage aufwerfen, wie im Rahmen einer verlagsgeschichtlichen Untersuchung - und aus kulturwissenschaftlicher Perspektive wäre dies hoch relevant - eine Geschichte der Exklusionen geschrieben werden könnte.

\section{III.2.8. Elena Poniatowska - verzögertes Interesse bei Suhrkamp}

Durch das weltliterarische Selektionsraster der internationalen Verlage und gatekeeper fallen auch, wie in Kap. II.2 bereits ausgeführt, Teile des Werkes der u.a. mit dem Cervantes-Preis ausgezeichneten mexikanischen Autorin Elena Poniatowska. Es drängt sich die Frage auf: Warum hat Suhrkamp, wo im Laufe der Jahre vier Werke der Autorin erschienen, nicht früher, nicht mehr und vor 
allem nicht die diskursbestimmenden Werke der Autorin verlegt? Aus den Marbacher Unterlagen des Siegfried Unseld Archivs geht hervor, dass der Literaturagent Mauricio Schoijet, der in Mexiko für Poniatowskas Verlag Era tätig war, Suhrkamp bereits 1971 in einem an Hans Magnus Enzensberger gerichteten Brief mehrere von Era publizierte Werke empfahl, darunter an erster und wichtigster Stelle La noche de Tlatelolco, einen damals noch jungen Klassiker der Testimonialliteratur über die blutige Niederschlagung der Studentenproteste am 2. Oktober 1968 in Mexiko-Stadt, die er als „große journalistische Collage“ (Brief von M. Schoijet an H. M. Enzensberger, 30.11.1971, SUA) bezeichnet. Schoijet betont den ungewöhnlichen Verkaufserfolg dieser journalistisch-literarischen Chronik in Mexiko, den er mit 50.000 verkauften Exemplaren beziffert. Zugleich reflektiert er nicht nur die zu erwartenden Schwierigkeiten bei der Übersetzung ins Deutsche, die in den vielen lokalen Bezügen und dem stark mexikanisch gefärbten Spanisch liegen, sondern er empfiehlt auch eine qualifizierte und bereits erprobte Übersetzerin, die in Mexiko-Stadt ansässig sei und das Werk im Austausch mit der Autorin übersetzen könne. Darüber hinaus bietet der Agent an, den Text mit einem auf die deutsche Leserschaft zugeschnittenen Anmerkungsapparat zu versehen und hat sogar einen Autor für ein potentielles Nachwort zu der politischen Lage in Mexiko organisiert (Brief von M. Schoijet an H. M. Enzensberger, 30.11.1971, SUA).

Im Verlag entschied man sich gegen die Veröffentlichung. In jenen Jahren publizierte Suhrkamp in seinem Lateinamerika-Programm beinahe ausschließlich Essays zu den linken Bewegungen und politischen Umbrüchen in Lateinamerika, von Peru 1965. Aufzeichnungen eines Guerilla-Aufstands (Héctor Béjar Rivera, 1970) über Venezuela. Die Gewalt als Voraussetzung der Freiheit (Orlando Araujo, 1971) bis Guatemala. Unterentwicklung und Gewalt (Juan Maestre Alfonso, 1971). Auch Darcy Ribeiros erstes von Suhrkamp verlegtes Werk, Der zivilisatorische Pro$z e ß$, kam 1971 heraus. Inhaltlich passt die Dokumentation von Poniatowska also hervorragend zu Suhrkamps Selektionskriterien für Werke lateinamerikanischer Autor/innen. Auch das Collagenhafte von La noche de Tlatelolco wird kaum als problematisch oder uninteressant aufgefasst worden sein, verstand sich Suhrkamp ja durchaus als literarisch progressiver Verlag. Allerdings gab es unter den lateinamerikanischen Suhrkamp-Autor/innen zu jenem Zeitpunkt, Anfang der 1970er Jahre, keine einzige Frau. Dies änderte sich erst 1978 mit einer Publikation der Brasilianerin Rachel de Queiroz, drei Jahre später gefolgt von Clarice Lispector (1981, 1982 und 1983). Die erste hispanoamerikanische Autorin, die Suhrkamp in sein Lateinamerika-Programm aufnahm, war 1984 Isabel Allende.

Was Elena Poniatowska anbelangt, begann der Verlag erst zu Beginn der 1980er Jahre, sich für ihre Arbeit zu interessieren. Ende 1980/Anfang $1981 \mathrm{schrieb}$ Michi Strausfeld, weiterhin entscheidend verantwortlich für das LateinamerikaProgramm bei Suhrkamp, Gutachten über zwei von Poniatowskas Werken: zum 
einen über den auf Interviews basierenden Testimonialroman über das ungewöhnliche Leben einer einfachen Mexikanerin, Hasta no verte, Jesús mío aus dem Jahr 1969, zum anderen über einen damals gerade erschienenen Band mit fünf Reportagen, betitelt Fuerte es el silencio. Ihr Fazit über Hasta no verte, Jesús mío:

\footnotetext{
Eine sehr gut geschriebene Roman-Biographie, mit der die Autorin sich zugleich als eine der wenigen guten weiblichen Schriftstellerinnen Lateinamerikas profiliert hat. [...] Da diese „Lebensberichte“ offensichtlich gutes Echo in Deutschland finden, würde ich die Publikation des Buches empfehlen - es zählt zu den besten seiner Gattung. (Gutachten von M. Strausfeld, 18.12.1980, SUA)
}

An der literarischen Qualität lässt Strausfeld keinen Zweifel und beendet ihr Gutachten mit einem Superlativ: Als Journalistin gelte Poniatowska als „,beste“ Lateinamerikas“ (ebd.). Dennoch passte auch dieser Roman offenbar nicht gut genug zu den Rezeptionsmustern des Suhrkamp Verlags. Dieses zweite große Werk, dem Poniatowska ihre internationale Bekanntheit verdankt und das auch ins Englische, Französische und Italienische übersetzt wurde, erschien stattdessen 1982 in dem kleinen Lamuv Verlag unter dem Titel Allem zum Trotz...: das Leben der Jesusa. Publiziert wurde bei Suhrkamp erst 1987 schließlich Stark ist das Schweigen. 4 Reportagen aus Mexiko, die Übersetzung von vier der fünf Reportagen aus Fuerte es el silencio (Ediciones Era 1980). Michi Strausfeld hatte sich bereits Anfang 1981 in ihrem Gutachten geradezu begeistert gezeigt:

Jedes Thema ist mitreissend geschrieben; literarische Reportagen, die aus den vielen, sorgfältig recherchierten Einzelheiten Geschichten zusammensetzen, die die mexikanische Realität eindringlich und plastisch vor Augen führt. Die Autorin hat wirklich ihre Stimme jenen Menschen geliehen, die nicht selber reden können: und sie schreibt so, dass man das Thema nicht wieder vergisst. [...] Ich empfehle die Publikation dieser Reportagen ganz dringlich! (Gutachten von M. Strausfeld, 19.01.1981, SUA)

Zwei Jahre nach Stark ist das Schweigen, 1989, entschloss man sich bei Suhrkamp, aus dem bereits vorliegenden Euvre ein weiteres Werk auf den deutschen Markt zu bringen. Es war aber nicht die Chronik des Tlatelolco-Massakers, die mit ihrem reportagehaften Stil perfekt an den Vorgänger angeschlossen hätte und zugleich ein für die linke Bewegung ikonisches Ereignis zum Thema hat; die Zeit der mit Lateinamerika verknüpften politischen Utopien hatte ihren Zenit längst überschritten. Die Wahl fiel auf Lieber Diego (Original: Querido Diego, te abraza Quiela, Ediciones Era 1978), einen Briefroman, der mit Diego Rivera ebenfalls eine mexikanische Ikone behandelt und ansonsten thematisch eher im Privaten und Emotionalen angesiedelt ist. Es folgten bei Suhrkamp Tinissima. Der Lebensroman der Tina Modotti (1996; Original: Tinísima, Ediciones Era 1992) und Frau des Windes (erschienen im Insel Verlag 2012; Original: Leonora, Seix Barral 2011). Bei der 
Auswahl des Verlagsprogramms spielt die politische, sozial engagierte Autorin Poniatowska eine eher untergeordnete Rolle; es überwiegt, vor allem bei den letzten drei Publikationen, ein Rezeptionsschema, das exotistische Inszenierungen mit einer dezidiert weiblichen Perspektive verbindet. Weder der Reportagenband noch die Romane sind allerdings auf der Seite des Suhrkamp Verlags als lieferbar gelistet (Stand: April 2019).

\section{III.2.9. Das Lateinamerika-Programm II: Die Phase nach dem Erfolg 2000-2017. Nach dem Exotismus}

Wie entwickelten sich die lateinamerikanischen Literaturen nach dem Boom, bevor sie in Deutschland ankamen? Wie bereits skizziert (Kap. II.1), kam es vereinzelt bereits im Spätwerk der Erfolgsautoren, ganz deutlich dann bei der jungen Autor/innen-Generation ab Beginn der 1990er Jahre zu einer ostentativen Abwendung von spezifisch lateinamerikanischen Themen. Wie reagiert Suhrkamp? Während das Lateinamerika-Programm in den 1980er und 1990er Jahren noch florierte, stagniert es seit 2000 deutlich. ${ }^{30}$ Zwischen den Jahren 2001 und 2017 lassen sich insgesamt ,nur' 105 neue Titel verzeichnen. In den 17 Jahren vor der Jahrtausendwende, von 1984-2000, waren es noch 164 Publikationen. Wenn man das statistische Jahresmittel der beiden Zeiträume gegeneinanderstellt, so hat sich die Zahl der jährlichen Veröffentlichungen aus Lateinamerika von etwa zehn Titeln pro Jahr vor der Jahrtausendwende auf sechs Titel pro Jahr nach der Jahrtausendwende fast halbiert.

Mit Blick auf Suhrkamps Publikationsstrategien ist der - notwendigerweise marktwirtschaftlich orientierte - Klappentext eines 2013 erschienenen Romans aus Lateinamerika aufschlussreich. Der Roman des aus Kolumbien stammenden, 1965 geborenen Schriftstellers Sergio Álvarez mit dem Titel 35 Tote wird folgendermaßen beworben:

Nach Hundert Jahre Einsamkeit - der große Kolumbien-Roman. Wer in diesem Land niemanden getötet hat, der hat keine Zukunft. Atemberaubend, erschütternd, fesselnd. Dem magischen Realismus von García Márquez' Hundert Jahre Einsamkeit setzt Sergio Álvarez mit 35 Tote einen kraftvollen Roman entgegen.

Inwiefern sind nun die literarischen Tendenzen der jungen Generation mit dem einstigen Anspruch an das Gütesiegel ,Weltliteratur' vereinbar? Was den Paradigmenwechsel in den Literatur- und Kulturwissenschaften von Weltliteratur zu

30 U.a. auch dieser Phase von Suhrkamps Lateinamerika-Programm widme ich mich ebenfalls in Müller (2014a, 2015a, 2015b). 
Literaturen der Welt betrifft (vgl. Kap. III.1), reagiert Suhrkamp getreu seines einstigen Anspruchs, mit aktuellen Tendenzen der intellektuellen Avantgarde im Dialog zu stehen und durch seine Autor/innenauswahl die Debatten maßgeblich zu bestimmen. So wirbt beispielsweise die Internetseite des Verlags für den osttürkischen Suhrkamp-Autor Nedim Gürsel, der 2011/2012 die Samuel Fischer Gastprofessur für Literatur: Literaturen der Welt an der Freien Universität Berlin innehatte. Suhrkamp nimmt den Anspruch der Denomination dieser Professur affirmativ auf und unterstreicht die Absicht des Verlags, „eine kritische Reflexion über die Literaturen der Welt zu fördern“. ${ }^{31}$ Denn diese bezieht sich auf Texte, die sich selbst in weltweiten Beziehungen verorten und in denen kulturelle Positionsbestimmungen reflektiert beziehungsweise ausgestaltet werden.

Selbstverständlich gibt es angesichts der Voraussetzungen transnationaler Lebensläufe und einer privilegierten Situation von nicht-sesshaften Literaturen gerade auch in Lateinamerika literarische Texte, die diese Lesarten ermöglichen. So beispielsweise einer der jüngsten Romane des langjährigen Suhrkamp-Autors Mario Vargas Llosa, El sueño del celta, erschienen 2010 beim spanischen Prestigeverlag Alfaguara. Darin finden transnationale Bewegungen zwischen drei Weltregionen - Irland, Kongo, brasilianischer Amazonas - statt. Doch Suhrkamp zeigte zunächst wenig Interesse: Als während der Frankfurter Buchmesse im Oktober 2010 die Vergabe des Nobelpreises für Literatur an Mario Vargas Llosa publik wurde (vgl. Meyer-Krentler 2010), feierte sich der Suhrkamp Verlag umgehend als deutschen Starverleger, während kurz darauf durchsickerte, dass der Verlag nur vier Wochen vorher bei Verhandlungen die deutsche Ausgabe des Sueño del celta abgelehnt hatte. Dafür hatte Rowohlt die Rechte bereits erworben. Die Entscheidung gegen den neusten Roman wurde im Kontext der Nobelpreisvergabe $z u$ einem Verlagsskandal, der sich über einige Monate hinzog, bis Rowohlt wieder auf die Rechte verzichtete und der Roman bei Suhrkamp unter dem Titel Der Traum des Kelten erscheinen konnte. Dies wurde im Herbst $2011 \mathrm{mit}$ einer Eröffnungsveranstaltung im Haus der Berliner Festspiele gefeiert. Die Eröffnungsrede der damaligen Verlagschefin Ulla Berkéwicz begrüßte den Roman als Rückkehr Vargas Llosas zu seinem ursprünglichen Erzählen, betonte die Andersartigkeit des spezifisch Peruanischen und die magische Erzählweise.

31 http://www.suhrkamp.de/news/samuel_fischer_gastprofessur_fuer_literatur_1702.html. 


\section{III.2.10. Samanta Schweblin - jüngste verlagspolitische Tendenzen}

Samanta Schweblin steht im Lateinamerika-Programm des Suhrkamp Verlags für jüngste verlagspolitische Tendenzen, die dahingehen, für die aktuelle Literatur des Subkontinents zwar eine globale Relevanz im Sinne eines universalistischen Anspruchs geltend zu machen, jedoch ohne besonderen Bezug zu spezifisch lateinamerikanischen Diskursen. Schweblin, die Mario Vargas Llosa „eine der vielversprechendsten Stimmen der modernen spanischsprachigen Literatur، ${ }^{32}$ nannte, wurde 1978 in Buenos Aires geboren. Sie hat bislang drei Erzählbände und zwei Romane veröffentlicht, die in 25 Sprachen übersetzt wurden (Literaturport). Ihre Kurzgeschichtensammlungen El núcleo del disturbio (2002), Pájaros en la boca (2008) und Siete casas vacías (2015) erhielten den Fondo Nacional de las Artes-Preis (Argentinien), den Premio Casa de las Américas (Kuba), den Juan Rulfo-Preis (Frankreich) und den hochdotierten Premio de narrativa breve Ribera del Duero (Spanien).Zudem stand die englische Übersetzung ihres ersten, 2014 erschienenen Romans Distancia de Rescate (Fever Dream) 2017 auf der Shortlist für den Man Booker International Prize (England) und gewann 2018 den Shirley Jackson Award (USA).

Die Rezensionen der internationalen Presse sparen nicht mit Superlativen: El País bezeichnet sie als „Meisterin der kleinen Form“ und sieht sie in einer Liga mit Jorge Luis Borges, Julio Cortázar oder Juan Rulfo, ${ }^{33}$ La Nación feiert Distancia

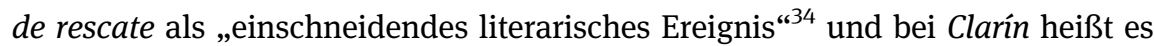
schlicht, Schweblin sei „la narradora argentina más prestigiosa, premiada y traducida del momento“ (Fernández 2018). Der Economist schreibt über Schweblins Debütroman Distancia de rescate, er beinhalte ein kompaktes, aber explosives Paket zeitgenössischer privater und öffentlicher Albträume („Samanta Schweblin“ 2017). In China, wo Schweblin zwei Monate im Rahmen des Shanghai Writers' Program lebte ${ }^{35}$ und der in Shanghai ansässige Verlag 99reader 2013 ihren Band Pájaros en la boca übersetzen ließ, wird ihre Literatur ähnlich positiv aufgenommen wie in der westlichen Welt. Schweblins Bildsprache ist in vielen Besprechungen herausgehoben worden. So kommentiert Daniel Alarcón, sie würde wie eine Lyrikerin über Bilder kommunizieren - wohingegen die chinesische Romanautorin A Yi Schweblins Geschichten sogar mit chinesischen Gemälden

32 Zit. nach https://www.suhrkamp.de/autoren/samanta_schweblin_8074.html.

33 Zit. nach https://www.suhrkamp.de/buecher/sieben_leere_haeuser-samanta_schweblin_428 04.html.

34 Vgl. https://www.suhrkamp.de/buecher/das_gift-samanta_schweblin_42503.html.

35 Vgl. https://de.wikipedia.org/wiki/Samanta_Schweblin. 
vergleicht: Sie sieht die Parallele in der Art, wie beide Kunstformen einen Raum für die Vorstellung des Lesers eröffnen (Xi 2013).

Das deutschsprachige Publikum konnte Samanta Schweblin 2010 erstmals entdecken. Zum Gastauftritt Argentiniens auf der Frankfurter Buchmesse ließ der Suhrkamp Verlag Schweblins zweiten Erzählband, Pájaros en la boca, übersetzen, gefördert durch den argentinischen Übersetzungsfond PROSUR. Seither erschienen bei Suhrkamp: Die Wahrheit über die Zukunft (2010; Original: Pájaros en la boca, 2008, übers. von Angelica Ammar), Das Gift (2015; Original: Distancia de Rescate, 2014, übers. von Marianne Gareis) sowie Sieben leere Häuser (2018; Original: Siete casas vacías, 2015, übers. von Marianne Gareis). Betrachtet man die Buchpräsentationen und die internationalen Pressestimmen, die auf der Homepage des Suhrkamp Verlages die Veröffentlichungen von Schweblin bewerben, so fällt auf, dass die Kritiken sich in ihrer großen Mehrheit auf die spezifische Schreibweise Schweblins fokussieren. So streicht Suhrkamp etwa den „meisterhaft lakonischen“36 Stil heraus und betont, Schweblin schaffe „einen eigenen, wundersamen Erzählkosmos“. ${ }^{37}$ Le Monde wiederum lobt die Autorin für ihren „einzigartigen Blick auf die Dinge“. ${ }^{38}$ Der bis vor kurzem bei lateinamerikanischen Autor/innen obligate Bezug zu deren Herkunft scheint bei Suhrkamp als Rezeptionsmuster nicht mehr an erster Stelle zu stehen, weder in Form einer literarischen Genealogie - wie etwa bei Vargas Llosa, dessen Roman El sueño del celta noch 2011 (fälschlicherweise) als Fortschreibung des Magischen Realismus gepriesen wurde - noch als Kampf und Abgrenzung gegen die Überväter der Boom-Generation - wie etwa bei Crack, McOndo oder, um bei Suhrkamp zu bleiben, bei Sergio Álvarez, dessen literarische Relevanz sich im Klappentext von 35 Tote aus dem Gegenentwurf zu García Márquez' Cien años de soledad ergab (vgl. Kap. III.2.9). Beinahe anachronistisch und merkwürdig zusammenhanglos, wie ein alter Reflex, wirkt es da, wenn in der Presse dann doch Schweblins „sprachliche Präzision“ gelobt wird, ,wie man sie nur ganz selten bei lateinamerikanischen Autoren antrifft“39 oder man die altbekannten Reizworte des Lateinamerika-Booms aufgereiht findet: „In Samanta Schweblins Argentinien wird das Magische politisch“ (Heidemann 2015).

36 https://www.suhrkamp.de/buecher/sieben_leere_haeuser-samanta_schweblin_42804.html. 37 https://www.suhrkamp.de/buecher/die_wahrheit_ueber_die_zukunft-samanta_schweblin_ 42142.html.

38 Zit. nach: https://www.suhrkamp.de/buecher/sieben_leere_haeuser-samanta_schweblin_42 804.html.

39 Tobias Wenzel vom WDR, zit. nach: https://www.suhrkamp.de/buecher/sieben_leere_haeu ser-samanta_schweblin_42804.html. Der ganze Artikel findet sich unter: https://www1.wdr.de/ kultur/buecher/sieben-leere-haeuser-104.html. 
Geht es um literarische oder ästhetische Verwandtschaften, so finden sich in Buchbesprechungen und Autorenportraits durchaus auch fundierte Bezüge zu einer argentinischen Literaturtradition, vor allem zu der Neophantastik eines Adolfo Bioy Casares, Julio Cortázar oder Jorge Luis Borges (vgl. z. B. Halter 2015; Müller, A. 2010). Dabei geht es natürlich auch um das Genre der Kurzgeschichte, das eine von Schweblins bevorzugten Ausdrucksformen ist und das in Argentinien eine große Tradition hat. Die Autorin selbst mag diese Zuschreibung indes nur eingeschränkt gelten lassen. Zum einen benennt sie selbst als ihre literarischen Vorbilder eher nordamerikanische Erzähler/innen wie Flannery O’Connor, John Cheever, Raymond Carver oder J.D. Salinger (Schweblin 2010; Quiroga 2015) und zum anderen betont sie, wie wichtig es ihr sei, dass ihre Literatur in der Schwebe bleibe zwischen dem Realen und dem Irrealen: „Die argentinische Literaturkritik sieht mich in der Tradition von Jorge Luis Borges oder Julio Cortázar. Das ist natürlich eine Ehre, ich bewundere ihre Werke. Aber ich glaube nicht, dass meine Geschichten zur fantastischen Literatur zählen. Denn sie könnten ja alle genau so geschehen!“ (Klobusiczky 2012). Treffender sind da vielleicht die Hinweise auf Franz Kafka oder auch auf David Lynchs halluzinatorische Bildwelten (vgl. Person 2010; Halter 2015; Heidemann 2015). Dabei handelt es sich bei Schweblin keineswegs um regional spezifische Formen etwa des „Magischen“, sondern ganz im Gegenteil um Themen und Perspektiven, die zugleich radikal individuell und von universeller Geltung sind, wie die Autorin mit Bezug auf Distancia de rescate selbst in einem Interview formuliert: „es una novela escrita desde la primera, primerísima persona, de punta a punta. Pasa en la cabeza de una mujer, y todo es personal. Y cuando todo es personal, se vuelve universal“ (Benavides 2018).

\section{III.2.11. Weltliterarische Konzepte in der Verlagswelt: aktuelle Perspektiven}

Es lässt sich festhalten, dass die Literaturen der Amerikas bis heute bei Suhrkamp durch den dominanten Rezeptionsfilter ,Weltliteratur sortiert werden. Dabei spielt - zumindest auf latente Weise - die Goethe'schen Provenienz des Konzepts eine Rolle. Symptomatisch ist die Einteilung des Verlags in regional getrennte Sparten. Diesen europäisch definierten Kriterien der Weltliteratur entsprachen die großen lateinamerikanischen Erfolgsromane. Von dem Moment an aber, wo sich die Autor/innen über diese Kriterien hinwegsetzten, verloren sie an Anerkennung. Der Rückgang des Lateinamerika-Booms bei Suhrkamp ist eine Antwort auf das Nicht-Einhalten eurozentrisch definierter Weltliteraturregeln, denen neben Universalismus auch Exotismus inhärent ist: Gerade die lateinamerikanischen Literaturen gewannen Anerkennung durch die Inszenierung eines exotistischen Identitätsdiskurses, der sich in Abgrenzung von einem europäischen 
Selbstverständnis definierte. In dem Moment, in dem die Romane die Inszenierung des spezifisch Lateinamerikanischen nicht mehr ins Zentrum stellten, büßten sie ihre weltliterarische Attraktivität ein und sind innerhalb des Suhrkamp'schen Verlagsprogramms nicht mehr von der zentralen Bedeutung, die ihnen zuvor zuteilwurde.

Wenn sich in den letzten zwanzig Jahren in Literaturwissenschaft und Literaturbetrieb vermehrt das Konzept der ,Literaturen der Welt' durchgesetzt hat, und auch einige Verlage und Kulturinstitutionen diesen Ausdruck aufgreifen, dann scheint Suhrkamp zwar auf den ersten Blick auf diesen Zug aufzuspringen, genauso unausgesprochen wie beim Konzept der, Weltliteratur', doch stellt sich bei näherem Hinsehen heraus, dass es sich dabei um mehr Schein als Sein handelt. Der Widerspruch zwischen plakativem Anspruch auf der Webseite einerseits und dem Fall Vargas Llosa im Jahre 2010 andererseits spricht für sich. Aufschlussreich für eine Beschäftigung mit der verlagspolitischen Programmatik sind gerade auch die Leerstellen im System, etwa die Nicht-Aufnahme William Faulkners und Édouard Glissants, oder auch bestimmter Werke Elena Poniatowskas, die es rein zeitlich gesehen - erlauben, einen Bogen $\mathrm{zu}$ spannen vom Beginn der Suhrkamp-Ära seit den 1950er Jahren bis heute. Das Beispiel Samanta Schweblin indes zeigt, dass aktuell auch eine Ablösung gelingt von den lang tradierten Erwartungen an lateinamerikanische Literaturen und den ihnen vermeintlich inhärenten exotistisch-essentialistischen Elementen. 